\title{
Ein Blick in die Geschichte der botanischen Morphologie und die Pericaulom-Theorie.
}

\author{
Von
}

\section{Dr. H. Potonié,}

Kgl. preuss. Landesgeologen u. Professor bezw. Privat-Dozenten der Palaeobotanik an der Kgl. Bergakademie und der Universität zu Berlin.

\author{
Erweiterter Abdruck \\ aus der \\ Naturwissenschaftlichen Wochenschrift N. F. II. Bd.j der ganzen \\ Reihe XVIII. Bd.
}

Mit 9 Abbildungen (in 6 Figuren).

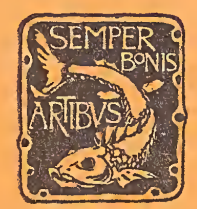

Jena.

- Verlag von Gustav Fischer. 1903. 


\section{Verlag von Gustav Fischer in Jena.}

Soeben wurde vollständig:

Chun, Carl, Aus den Tiefen des Weltmeeres. Schilderungen von der deutschen Tiefsee-Expedition. Mit 6 Chromolithographien; 8 Heliogravüren, 32 als Tafeln gedruckten Vollbildern, 2 Karten und 482 Abbildungen im Text. Zweite, umgearbeitete und durch nouo Abbildungen vermehrte Auflage. Preis des vollständigen Werkes: broschiert M. 18:-, elegant gebunden M. 20.-. Ausführliche Prospekte durch jede Buchhandlung zu erhalten.

Münchener Neueste Nachrichten Nr. 18, 11. Januar 1901 über die 1.Aufl.:

Die von uns wiederholt empfohlenen Schilderungen von der deutschen Tiefsee-Expedition unter Leitung des Professors Dr. C a rl C h un sind jetzt mit dem Erscheinen der letzten Lieferungen abgeschlossen. Dem Werk ist ein vortreffliches Register beigegeben worden; eine ges chmackvolle Einb anddecke ist zum Preise von 1 Mk. 60 Pf. zu beziehen. - Über das monumentale Werk selbst können wir uns jetzt ganz kurz fassen: es ist einer der vornehmsten und hervorragendsten Beiträge zur gemeinverständlichen wissenschaftlichen Litteratur, ein glänzendes Zeugnis deutscher Forschertüchtigkeit, ein bleibendes wertvolles Denkmal einer ergebnisreichen Forschungsreise - ein wahres Prachtwerkfür das deutsche Volk. Die Verlagsbuchhandlung hat sich durch die prachtivolle Ausstattung mit den zahllosen hochinteressanten Illustrationen ein ausserordentliches Verdienst erworben. Der Preis des Werkes ermöglicht eine weite Verbreitung, die wir dem Chunschen Buche aufrichtig wünschen.

\section{Mitteilungen, botanische, aus den Tropen, her- ausgegeben von Dr. A. F. W. Schimper,} o. ö. Professor der Botanik an der Universität Bonn. 9 Hefte. 1888-1901. Lex.-Form. Preis: M. 109.-.

Heft 1: Schimp er, A. F. W., Die Wechselbeziehungen zwischen Pflanzen und Ameisen im tropischen Amerika. 1888. Mit 3 Tafeln. Preis: M. 4.50.

Heft 2: Schimper, A. F. W., Die epiphytische Vegetation Amerikas. Mit 6 Tafeln. 1888. Preis : M. 7.50.

Heft 3: Schimper, A. F. W., Dio indo-malayische Strandflora. Mit 7 Textfiguren, einer Karte und 7 Tafeln. 1891. Preis: III. $10 .-$.

Heft 4: Schenck, Dr. H., Privatdocent an der Universität Bonn, Beiträge zur Biologie und Anatomie der Lianen, im besonderen der in Brasilien einheimischen Arten. I. Teil: Beiträge zur Biologie der Lianen. Mit 7 Tafeln. 1892. Preis: II. 15 . -

Heft 5: S chenck, H., Beiträge zur Biologie und Anatomie der Lianen, im besonderen der in Brasilien einheimischen Arten. II. Teil: Beiträge zur Anatomie der Lianen. Mit 12 Tafeln und 2 Text-Zinkograph. 1893. Preis: M. 20.-.

Heft 6: Möller, A lf red, Die Pilzgärten einiger amorikanischer Ameisen. Mit 7 Tafeln und 4 Holzschnitten. 1893. Preis M. 7.-.

Heft 7: Möller, Alfred, Brasilianische Pilzblumen. Mit 8 Tafeln. 1895. Preis: M. 11.-

Heft 8: Möller, Alfred, Protobasidiomycoton. Untersuchungen aus Brasilien. Mit 6 Tafeln. 1895. Preis : M. 10.-

Heft 9: Möller, A lfred, Phycomyceten und Ascomyceten. Untersuchungen aus Brasilien. Mit 11 Tafeln und 2 Textabbildungen. 1901. Preis: M: 24.- 


\section{Ein Blick in die Geschichte der botanischen Morphologie und die Pericaulom-Theorie.}

Von

\section{Dr. H. Potonié,}

Kgl. preuss. Landesgeologen u. Professor bezw. Privat-Dozenten der Palaeobotanik an der Kgl. Bergakademie und der Universität zu Berlin.

\section{Erweiterter Abdruck}

aus der

Naturwissenschaftlichen Wochenschrift N. F. II. Bd., der ganzen Reihe XVIII. Bd.

Mit 9 Abbildungen (in 6 Figuren).

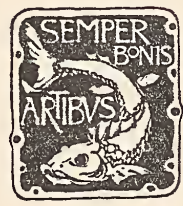

Jena.

Verlag von Gustav Fischer. I 903. 
QE905

. P58

1912 


\section{Vorwort.}

Die vorliegende Abhandlung ist ein Wiederabdruck von zwei zusammengehörigen aber aus bestimmten Gründen getrennt gebrachten Veröffentlichungen, die unter den Titeln:

I. Ein Blick in die Geschichte der botanischen Morphologie mit besonderer Rücksicht auf die PericaulomTheorie. (Naturwissenschaftliche Wochenschrift. Jena 5.-19. Oktober 1902), und

2. Die Pericaulom-Theorie (Berichte der Deutschen Botanischen Gesellschaft. Berlin, Sitzung vom 3I. Oktober 1902)

erschienen sind.

Dementsprechend mussten beide Artikel vorliegend durch Veränderungen im Text einander angepasst werden; ferner wurde die Gelegenheit benutzt hier und da zu korrigieren und etwas zu erweitern.

Gr.-Lichterfelde-West bei Berlin im November 1902.

H. Potonié. 

Das erste Bedürfnis, das sich der Lebewelt gegenüber naturgemäss geltend macht, ist dies: in der zunächst verwirrenden Mannigfaltigkeit der entgegentretenden Gestaltungen aus praktischen Rücksichten eine Uebersicht zu gewinnen. Das ist nur möglich, wenn man den Versuch macht, in dieselbe Ordnung zu bringen, d. h. sie in bestimmter Weise durch Aufsuchung von Gemeinsamkeiten zu gruppieren, zu klassifizieren, begrifflich zusammenzufassen. Aus diesem Bedürfnis heraus sind die Systeme des Tier- und Pflanzenreichs entstanden.

Aber nicht nur die Individuen wollen klassifiziert sein, sondern auch die Fülle der Teile, die Organe, die die Individuen zusammensetzen.

Als Gesichtspunkt, nach welchem dies geschah, konnte ursprünglich - da sich die Anknüpfung an das dem Menschen zunächst liegende von selbst ergab, nämlich an den Menschen selbst - kein anderer in Betracht kommen als derjenige, der die Beziehungen der Organe zur Aussenwelt betrachtet, mit anderen Worten: das erste Prinzip der Einteilung und dementsprechend Benennung der Organe ist ein physiologisches; denn es ist die stillschweigende Annahme des Volkes, wenn es die von ihm geschaffenen Begriffe Wurzel, Stengel, Blatt, Blüte u. dergl. ausspricht, dass die Verschiedenheiten, die diese Worte zum Ausdruck bringen sollen, solche physiologischer Art sind. Es verknüpft sich beim Laien unfehlbar mit dem Gebrauch irgend eines derselben der Gedanke an die Thätigkeit (die Funktion), die Bedeutung der Organe für das Leben der Pflanze.

Die erste eingehendere, wissenschaftliche Beschäftigung mit den Pflanzen konnte daher nur von der physiologischen Einteilung der Organe ausgehen: wir haben als erste wissenschaftliche Periode der Beschreibung der Organe (der Organographie) die naive physiologische Periode. 
Freilich konnte man mit dieser Betrachtungsweise, die für das Tierreich durch seine nahe Beziehung zum Menschen immer auffällig hervorgetreten ist, bei den Pflanzen nicht weit kommen, da es zunächst an hinreichenden Kenntnissen über die Funktionen der Pflanzenorgane fehlte. Es verlief sich daher die botanische Wissenschaft in eine lange Zeit hindurch herrschende Beschreibung der äusseren und inneren Gestaltungsverhältnisse, der blossen geometrischen Formen der Organe, ohne diese Formen weiter in $\mathrm{Zu}$ sammenhang bringen zu können. Es ist das die Periode der sogen. beschreibenden Botanik, „sogenannt" weil der Zusatz „,beschreibend“ insofern unpassend gewählt ist, als die Wissenschaft über Beschreibungen überhaupt nicht hinauszukommen vermag, nur freilich mit dem Unterschiede, dass die Botanik der beschreibenden Periode im wesentlichen über die Betrachtung der Einzelheiten nicht hinauskam, während die jetzige Wissenschaft die Beschreibung der Beziehungen, der Zusammenhänge, als das Wichtigere erkannt hat.

Die lange Dauer der beschreibenden Periode wurde durch das Erfordernis begünstigt, die zahlreichen zur Kenntnis gelangenden Pflanzenarten schnell zu "bearbeiten“, d. h. im "System“, das ursprünglich weiter nichts als ein nach bestimmten praktischen Prinzipien geordneter Katalog war, unterzubringen, um sie bequem wiederfinden zu können.

Aber schon von vornherein haben einzel ne hervorragende Erforscher der Lebewesen (Biologen) mehr oder minder klar bemerkt, dass gewisse Organe verschiedener Funktion doch in gewissen Punkten auffällige organographische A.ehnlichkeiten aufweisen, eine Thatsache, die auf einen tieferen Grund hinwies, dessen Erforschung sich aufdrängte. Gerade diese Einsicht in Verbindung mit der auffälligen Mannigfaltigkeit z. B. der Blätter ist es, welche überhaupt geistreichen Beschauern der Natur ein Problem gesetzt hat, nicht minder wie der so variable und doch nach bestimmten "Typen" sich darstellende Gesamtbau der Pflanzen (und Organismen überhaupt). So spricht z. B. J. J. R o uss e a u von der unwandelbaren Aehnlichkeit und doch so wunderbaren Verschiedenheit, die in der Organisation der Pflanzen herrsche, und Goethe's vielcitierte Verse: „Alle Gestalten sind ähnlich, und keine gleichet der anderen; Und so deutet das Chor auf ein geheimes Gesetz," drücken dasselbe mit anderen Worten aus.

Die Bemühung diese gestaltlichen Beziehungen, die die Organismen in besonderer Weise miteinander verknüpfen, aufzusuchen und festzulegen hat eine Disziplin 
geschaffen, die zu dem I8I 7 von Goethe vorgeschlagenen Namen Morphologie geführt hat.

Danach wäre - sofern man, wie ich das in dem vorliegenden Fall für zweckmässig halte, die ursprünglichen Begriffsbestimmungen festhalten will - scharf $\mathrm{zu}$ unterscheiden zwischen Organographie *) und Morphologie. Es soll also im folgenden stets nur dann von morphologischen Eigentümlichkeiten die Rede sein, wenn es sich um theoretische Erörterungen handelt, die sich aus der vergleichenden Betrachtung der gestaltlichen Verhältnisse der Organismen ergeben, während die Organographie sich ganz allgemein - wenigstens der ursprünglichen Fassung gemäss - mit den Gestaltungsverhältnissen und ihrer praktischen Rubrizierung beschäftigt, ohne dabei theoretische Erwägungen anzustellen.

Es muss dies ja besonders betont werden, weil der genauen Uebersetzung unseres Terminus gemäss heutzutage unter Morphologie ganz allgemein auch einfach die Betrachtung der Gestaltungsverhältnisse, der Formen der jeweilig berücksichtigten Objekte verstanden wird, gleichgültig ob diese der Natur oder menschlicher Thätigkeit entstammen; so hat man sich denn gewöhnt, auch von der Morphologie der Krystalle u. s. w. zu reden. Es handelt sich also hier um die blosse Einzelbeschreibung der Formen der Einzelobjekte, und eine Hervorkehrung „morphologischer" Beziehungen bedeutet hier weiter nichts als eine Bezugnahme auf formale Aehnlichkeiten. Um ein besonderes Beispiel aus der Botanik zu erwähnen noch die folgende Bemerkung.

Wenn De Bary von der "Morphologie" eines Pilzes spricht, so meint er damit ausschliesslich die auf den Bau bezüglichen Verhältnisse desselben; spricht jedoch ein Botaniker aus der Schule A lexander Braun's von der "morphologischen Natur" eines bestimmten Organes, so will er, wie Goethe, die von ihm an die Betrachtung der Gestaltungen geknüpften theoretischen Erörterungen besonderer Art als das Wesentliche seiner Untersuchung angesehen wissen. Man versteht also unter Morphologie zweierlei. Beschränken wir den Sinn des Begriffs Morphologie (wenigstens in den biologischen Disziplinen) wieder auf die ursprüngliche Fassung desselben, so wäre der leider immer mehr in den Hintergrund gedrängte Terminus Organographie zur Be-

\footnotetext{
*) Gelegentlich wird auch der Ausdruck Organologie gebraucht; auch „Organogenie" für den Teil der Organographie, der sich insbeson dere mit der Entwicklung der Organe beschäftigt, war früher hier und da gebräuchlich und taucht jetzt wieder häufiger auf.
} 
zeichnung der Disziplin, die sich nur und ausschliesslich mit der Beschreibung des mit den Sinnen Konstatierbaren an den einzelnen Organen, der formalen Bestandteile der Lebewesen beschäftigt, in der That am Platze. Ein Buchtitel wie "Organographie végétale" (ich denke dabei an das Werk Aug. Pyr. de Candolle's von i827) ist klar und bringt keinerlei Zweifel bezüglich des Inhaltes. Es ist bedauerlich, dass heute die Unterscheidung in Organographie und Morphologie nicht mehr genügend festgehalten wird; noch Aug. de Saint-Hilaire sagt zur Auseinanderhaltung beider treffend von der Morphologie *), sie sei „l'organographie expliquée par les transformations auxquelles sont soumises les parties des végétaux".

Heutzutage ist also der Begriff Morphologie vollständig verwässert worden: er wird pleonastisch gebraucht ***) und als gänzlich überflüssiger Zusatz. ***) Da diese Begriffsverschiebung aber in diesem Falle blosser Oberflächlichkeit zuzuschreiben ist und keineswegs einem dringenden Bedürfnis entspringt, so werden wir in der Anwendung des Wortes Morphologie nicht dem allgemeinen heutigen Gebrauch folgen, sondern dasselbe vielmehr nur sinngemäss anwenden.

Von vornherein hat sich den Autoren bei der Betrachtung morphologischer Beziehungen der Gedanke aufgedrängt, dass sich dieselben durch einen tiefer liegenden Grund erklären; heute erblicken wir denselben in der wohlverstandenen $\dagger$ ) Phylogenesis der Lebewesen.

*) Leçons de botanique comprenant principalement la morphologie végétale. Paris 1840, p. I7.

**) So kann man z. B. oft von dem "morphologischen Bau" der Zelle u. dergl. lesen und hören.

***) Man vergleiche z. B. die Bezeichnung ,Morphologie der Erdoberfläche".

$\dagger$ Das heisst: Es kann natürlich nicht davon die Rede sein, dass zwei heutige Lebeformen mit Organen, die morphologisch aufeinander bezogen werden, sich nun ohne weiteres dadurch als von einander abstammend ergeben. Vielmehr liegt es weit näher, die morphologische Vergleichbarkeit durch die Herkunft der Formen von gemeinsamen Vorfahren zu erklären.

$\mathrm{Da}$ gleiche Bedingungen gleiche Folgen haben, so ist es für unsere Betrachtung gleichgültig, ob wir einen mono- oder - wie das richtiger sein dürfte - einen polyphyletischen Stammbaum annehmen, ob wir also der Anschauung huldigen, dass alle Organismen untereinander blutsverwandt sind oder nicht. Namentlich $\mathrm{N}$ äg eli (Abstammungslehre 1884) hat darauf aufmerksam gemacht, dass es unseren sonstigen Kenntnissen besser entspricht, Urzeugung an vielen Stellen gleichzeitig und vielleicht auch zu den verschiedensten Zeiten anzunehmen, sodass das Vorhandensein vieler nicht blutsverwandter Organismenreihen wahrscheinlicher ist. Mit Anlehnung an die Sprechweise der philosophischen Schule von Rich. Avenarius würde ich sagen: die Ergänzung, die die Descendenztheorie zu den Erfahrungsthatsachen versucht, ist diesen 
So sagt denn auch C. Nägeli (Abstammungslehre S. 46I) mit Fug und Recht:

„Ich hebe . . . ausdrücklich hervor, dass . . . die Bedeutung einer jeden vererbten, physiologischen oder morphologischen Erscheinung im Bauplan des ganzen Pflanzenreichs nur auf dem phylogenetischen Wege erforscht werden kann." Oder mit anderen Worten (1. c. p. 457-458): „Wir kommen ... immer zu dem Satze, dass nur die phylogenetische Einsicht uns über die Bedeutung der organischen Einrichtungen und ihre Stellung im ganzen Bauplan der organischen Natur Auskunft zu geben vermag." Die älteren Botaniker aber, denen G o e th e I790 folgte, erklärten sich die in Rede stehenden Beziehungen durch „Metamorphose“, sodass sich die Morphologie auch als Metamorphosenlehre bezeichnet findet. Was damit gemeint war, wird näher zu erläutern sein.

Wenn aber auch Botaniker von Fach schon vor dem grossen Dichter Anläufe genommen haben, so hat doch erst seine im angegebenen Jahre erschienene Schrift über den „Versuch die Metamorphose der Pflanzen zu erklären" in den Fachkreisen Eindruck gemacht: diese hat also zur Entwicklung der Morphologie den Hauptanstoss gegeben.

Eine zweite Etappe knüpft sich an den Namen Alexander Braun's. Um den Gesichtspunkt, der die

homogener, wenn die Nägeli'sche Annahme gemacht wird, während es eine heterogene Ergänzung wäre, sich vorzustellen, dass nur ein einziges Mal die Umstände für die Bildung von Lebewesen vorhanden gewesen sei. $\mathrm{Da}$ aber die Aenderungsbedingungen (die inneren und die äusseren) für die Organismen die gleichen oder doch sehr ähnliche sind, so werden die Ausgestaltungen der Organismenreihen doch eng vergleichbare

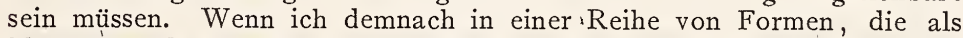
blutsverwandt gelten müssen, begründen kann, dass z. B. die Samen der höheren Pflanzen aus Sporangien hervorgegangen sind, so werde ich dasselbe für Parallelreihen annehmen können, und ich werde sogar wenn ich es nicht anders habe - in der Lage sein, die verschiedenen Etappen verschiedener Reihen heranzuziehen, um die morphologische Herkunft komplizierterer Organe klarzulegen. Habe ich eine Reihe, die mir die Annahme aufdrängt, dass aus der Fächer-(Parallel-)Aderung in den folgenden Generationen zunächst die Fieder-Aderung, sodann die einfache und endlich die Doppel-Maschen-Aderung hervorgegangen ist, so werde ich in homogener Ergänzung Aehnliches in anderen Reihen annehmen müssen, die wegen noch nicht aufgefundener Uebergangsglieder diesbezügliche Lücken aufweisen. Es ist dabei zu beachten, dass infolge der zeitlich' wiederholten Urzeugung neue Reihen wiederholt eingesetzt haben, von denen ein Teil durch die einfacheren Verhältnisse hindurch erst allmählich zu den höheren Komplikationen in ihrem Bau gelangen, sodass wir vielfach in den verschiedensten Zeiten gleichzeitig die verschiedenen Komplikationen nebeneinander vorhanden sehen. Auch aus ein und demselben Stammbaum können ältere Typen neben neu entstandenen bestehen bleiben. 
Goethe bis Braun'sche Morphologie leitet, von vornherein zu kennzeichnen ist die Nebeneinanderstellung der Spezialbegriffe, die mit dem Worte Metamorphose verknüpft werden, geboten.

Im ältesten Sinne, sagen wir in demjenigen Ovid's, wäre eine Metamorphose die plötzliche Verwandlung, wie diejenige Jupiters in einen Schwan, ein Begriff, den ein Naturforscher nicht gebrauchen kann, da sich solche mythischen Umwandlungen nicht beobachten lassen. Die übliche Benutzung des Wortes Metamorphose auf zoologischem Gebiet für die Thatsache, dass häufig, wie z. B. bei den Insekten, die Jungen vom Muttertiere getrennt auffällige Entwicklungsstadien durchmachen, ehe sie der Mutter ähnlich werden, ist ebenso allgemein bekannt. Auf Pflanzen angewendet bedeutet Metamorphose bei der Goethe-Braun'sche Schule die Mannigfaltigkeit, in der uns die "Ideen" im Sinne P lato's sinnlich entgegentreten. Die Schule sucht mehr unbewusst als bewusst diese Ideen $\mathrm{zu}$ finden, besonders die Idee der Wurzel, des Stengels und des Blattes. Hier haben wir es demnach mit Metaphysik zu thun, vor der sich die Naturwissenschaft zu hüten hat. Setzen wir an Stelle der gesuchten "Id e e n“ die realen Wurzeln, Stengel und Blätter und diejenigen Organe, aus denen sich die Wurzeln, Stengel und Blätter der höher differenzierten Pflanzen im Verlaufe der Descendenz der Lebewesen herausgebildet haben, so haben wir den heute einzig zulässigen Sinn des Begriffes Metamorphose auf morphologischem Gebiete. Die Metamorphosenlehre hat danach die Veränderungen klarzu legen, welche die Organe im Verlaufe der Generationen erlitten haben: hat die morphogenetische*) Herkunft der Organe festzustellen. Das wird zwar prinzipiell anerkannt und doch gilt immer noch Nägeli's Wort: „Man beschäftigt sich viel mit der Abstammung der Pflanzensippen, aber nicht mit der Herleitung der einzelnen Organe und Teile der Pflanzen, und doch muss diese vorausgehen und den Boden für jene bereiten."

Um die Gegensätze noch klarer hervortreten zu lassen und die Entwicklung der Disziplin eingehender $\mathrm{zu}$ verfolgen, sei ein knapper historischer Ueberblick gegeben.

Es ist speziell die ausserordentliche Mannigfaltigkeit

*) Der Ausdruck Phylogenie bezieht sich auf die Stammesgeschichte der Species etc.; betrachten wir jedoch besondere Organteile oder besondere Organe hinsichtlich ihrer Umbildungen im Laufe der Generationen, so ist der umfassendere Ausdruck Morphogenie besser am Platze. Morphogenie und Morphologie decken sich also ihrem Sinne nach. 
der Blätter, die zu unübersehbar häufiger Beschäftigung mit denselben Veranlassung gegeben hat, und von ihrer Betrachtung gehen auch die ersten Gedanken zur Morphologie aus.

Schon im I6. Jahrhundert hatte Andrea Cesalpini die Blumenkrone schlechtweg als folium bezeichnet*) um dadurch gewisse Uebereinstimmungen zwischen ersterer und dem Laubblatt zum Ausdruck zu bringen.

Im 17. Jahrhundert begegnen wir **) Andeutungen nach dieser Richtung bei Joachim Jung 1678 und Marcellus Malpighi 1687.

Im I8. Jahrhundert ist ***) Chr. G. Ludwig I742 zu nennen, der z. B. von den Bracteen, Nebenblättern, Ranken, Dornen, Haaren und Drüsen sagt, dass sie den Blättern analog seien, dass Filamente öfter in Petala verwandelt würden u. s. w.

Die zweite Hälfte des I8. Jahrhundert hat aber erst den eigentlichen Grund zur Morphologie gelegt.

Zuerst ist hier Linné zu nennen, der die so naheliegende Zusammenfassung der Anhangsorgane des Stengels als „Blätter" I 755 durch den Satz hervorhebt: „principium florum et foliorum idem est". Schon $175 \mathrm{I} \dagger$ ) hat er Gedanken geäussert, die in dieser Richtung liegen.

Der citierte Linné'sche Satz kann gewissermassen als Motto der ganzen „Metamorphosenlehre" gelten, da sie von dem in demselben ausgesprochenen Gedanken ausgeht; denn immer sind es die Blätter der Pflanzen, die zunächst als Objekte der 'morphologischen Forschung vorgenommen werden, und es ist ja bei der ausserordentlichen Augenfälligkeit und Wichtigkeit derselben ohne weiteres verständlich, dass eine wissenschaftliche Beschäftigung mit der Pflanzenwelt gerade diese Organe stets in eine ganz hervorragende Betrachtung gerückt hat. So lange die organographische, dann die morphologische Richtung herrschte, war es die Mannigfaltigkeit in der Entwicklung, Ausbildungsweise und dem Auftreten, welche zu erschöpfen gesucht wurde; die Physiologie hat dann die vielen Funktionen, welche das Blatt haben kann, klargelegt.

Die als Metamorphosis von Linné I 755 gelehrte Theorie war kurz die folgende. ††) Linné sucht die kon-

*) Vergl. S a ch s, Histoire de la botanique. Paris 1892 . p. I63 ff.

*) Nach Wi eg a nd, Kritik u. Gesch. d. Lehre v. d. Metam. d. Pfl. 1846 p. $24-25$.

****) Ebenfalls nach Wiegand p. 27.

†) Vergl. Wi e gand 1846 p. $27 \mathrm{ff}$.

†f) Nach Ascherson, Forskal üb. die Metam. d. Pfl. - Ber. d. D. bot. Ges. 1884 p. 294. 
zentrischen Blattkreise der Blüte mit den ebenfalls konzentrischen Gewebezonen des Stammes in genetische Beziehung zu bringen: den Kelch mit der Rinde, die Blumenkrone mit dem Phloëm, die Staubgefässe mit dem Holze, den Fruchtknoten mit dem Marke, und er vergleicht die Entfaltung der Blüte, in der diese im Stengel verborgenen Elemente aufällig zu Tage treten sollen, mit der Metamorphose des Schmetterlings aus der unscheinbaren Raupe.

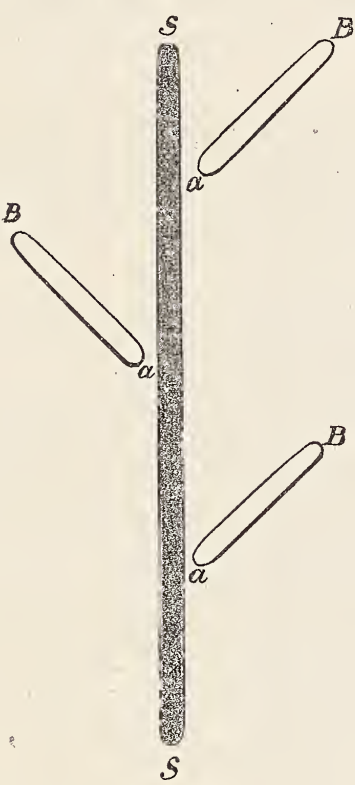

Fig. I. Schema des Aufbaus der höh. Pflanzen nach Cas par Friedrich Wolff. $\mathrm{S}=$ Stengel, $\mathrm{B}=$ Blätter, $\mathrm{a}=\mathrm{An}$ satzstellen der letzteren an dem Stengel.

Die Prolepsis Linné's, I760, erklärt dieselben Blattkreise *) im Sinne der damals herrschenden Evolutionstheorie ** für Produktionen mehrerer von einander abstammender, eigentlich für ebensoviel Jahrgänge bestimmter, aber für die Erfüllung des Fortpflanzungszwecks vorweggenom men e r Sprossgenerationen. Dieser Auffassung liegt nun die Annahme zu Grunde, dass die die Blüte zusammensetzenden Blätter aus Laubblättern hervorgehen.

Vorläufer der Prolepsistheorie sind $\mathrm{Caesalpin}$ und Malpighi, später, d. h. nach Linné, ist sie von $\mathrm{S} c h \mathrm{miedel}$ I 783 vertreten worden.

Weiter ist CasparFriedrich Wolff zu nennen. Dieser hat I759 die Stengelorgane und Blätter als unvereinbar gegensätzlich gedacht, also der Volksanschauung gehuldigt; aber er war zu dieser Ueberzeugung durch exakte entwicklungsgeschichtliche Thatsachen gelangt, die ihm die Blätter seiner Untersuchungsobjekte als strikte Seitenorgane erkennen liessen: hat er doch bei Brassica bereits den Vegetationspunkt gesehen und als solchen erkannt. Als Schema von Wolff's Ansicht mag Fig. I

*) 1. c.

**) Die Evolutionstheorie lehrte: Alle einzelnen Lebewesen sind vollständig fertig vorgebildet in einander geschachtelt (daher Einschachtelungstheorie) seit Anbeginn vorhanden; eine Neuentstehung in den elterlichen Individuen wurde abgelehnt.

***) Wi g gand I846 p. 33 . 
dienen: $s$ ist das Stengelorgan, $b$ sind die Blätter. Die Lücken an den Ansatzstellen der letzteren sollen daran erinnern, dass nach dieser Ansicht Stengel und Blätter Stücke für sich sind.

Peter Forskal hat I775 ebenfalls*) und zwar auf Grund der Beobachtung vergrünter Blüten die Blätter derselben mit denen der Laubblätter in dieselbe Kategorie gebracht. Den Satz Forskal's „flos est compendium tantae caulis massae, quantae foliorum habet", auf den Ascherson (1. c. p. 295) aufmerksam macht, übersetzt dieser in neuzeitlich botanische Sprache wie folgt: „die Blüte ist eine Zusammendrängung einer Anzahl Internodien mit den $\mathrm{zu}$ ihnen gehörigen Blättern, ein beblätterter Spross".

Wir kehren nun zu Goethe zurück. - Ein Goethe konnte als Naturforscher bei der Betrachtung der Einzelheiten nicht stehen bleiben; ein lexikographisches Wissen ist wohl als einziges Mittel zum Zweck einer Erkenntnis der Zusammenhänge im Weltganzen zu verlangen, vermag aber nur denjenigen für sich allein zu befriedigen, dem die Natur den nicht zu verlöschenden Drang versagt hat, das „Wesentliche" zu suchen: „den ruhenden Pol in der Erscheinungen Flucht".

Diese Eigenart eines echten Forschers bekundet sich ganz besonders in Goethe's morphologischen Studien.

In seiner Sammelschrift von i 8 i 7 "Zur Morphologie", die auch seinen „Versuch, die Metamorphose der Pflanzen zu erklären" von "I790 in Wiederabdruck enthält, sagt er: „Es hat sich ... in dem wissenschaftlichen Menschen $\mathrm{zu}$ allen Zeiten ein Trieb hervorgethan, die lebendigen Bildungen als solche zu erkennen, ihre äusseren, sichtbaren, greiflichen Teile im Zusammenhange $\mathrm{zu}$ erfassen, sie als Andeutungen des Innern aufzunehmen und so das Ganze in der Anschauung. gewissermassen zu beherrschen. - Man findet daher in dem Gange der Kunst, des Wissens und der Wissenschaft mehrere Versuche, eine Lehre zu gründen und auszubilden, welche wir die Morphologie nennen möchten." - Um noch besser zu zeigen, was Goethe unter Morphologie verstand, seien auch die ferneren Sätze citiert: „Betrachten wir aber alle Gestalten, besonders die organischen, so finden wir, dass nirgends ein Bestehendes, nirgends ein Ruhendes, ein Abgeschlossenes vorkommt, sondern dass vielmehr alles in einer steten Bewegung schwanke. Daher unsere Sprache das Wort.

*) Vergl. Aschers on 1884 . 
Bildung sowohl von dem Hervorgebrachten als von dem Hervorgebrachtwerdenden gehörig genug zu brauchen pflegt. - Wollen wir also eine Morphologie einleiten, so dürfen wir nicht von Gestalt sprechen, sondern, wenn wir das Wort brauchen, uns allenfalls dabei nur die Idee, den Begriff oder ein in der Erfahrung nur für den Augenblick Festgehaltenes denken."

Was den theoretischen Inhalt der Morphologie, das Problem derselben betrifft, so ist, wie wir aus den citierten Sätzen ersehen, bei Goethe, der mehr einem Ahnungsgefühl folgte, ohne sich zu voller Klarheit durchzuringen, bei unserer auf naturwissenschaftlichem Gebiet mit Recht allem Metaphysischen abgeneigten Forschung nichts uns heute Befriedigendes zu entnehmen. Er hat seine Ansichten in der schon citierten Abhandlung über die Metamorphose der Pflanzen niedergelegt, welche sich im wesentlichen mit den Blättern der Pflanzen beschäftigt.

Goethe sieht im Gegensatz zu Wolff die Pflanze aus lauter einheitlichen Stücken zusammengesetzt. Ein Spross besteht nach ihm - wie das Schema Fig. 2 veranschaulichen soll - im Prinzip aus Stengelstücken, die oben je ein Blatt tragen; je ein Stengelstück und ein Blatt. gehören als eine Einheit zusammen: in dieser glaubt er das $\mathrm{x}$ gefunden $\mathrm{zu}$ haben.

„Die Pflanze - sagt Goethe*) - stellt die verschiedensten Gestalten durch Modifikationen eines einzigen Organs dar."

Von den Ansichten Goethe's und seiner Vorgänger ist nun freilich für die Folge nur diejenige bei der Alltagsbotanik wirksam geworden und auch diese nur zaghaft und sehr langsam, die darauf hinauslief, die als Laubblätter, Kelch-, Kronenblätter, Staubgefässe u. s. w. bezeichneten Anhangsorgane der Stengelteile alle als „Blätter" begrifflich zusammenzufassen, da sie hierzu genügend Gemeinsamkeiten aufweisen, wie ihre Stellung, ihre unter Umständen gegenseitige Ersetzbarkeit; das Vorkommen von Blättern, die zum Teil z. B. laubblattartig, zum. Teil kronenblattartig ausgebildet sein können, die von Wolff zuerst nachgewiesene Uebereinstimmung ihrer Entstehung u. s. w. Die weitere wichtige Frage, woher denn nun diese Gemeinsamkeiten kommen, wie diese sich erklären, wurde in der Folgezeit kaum zu beantworten versucht; dabei hatte Goethe in seinem gesunden Denken und Fühlen, wie u. a. aus den eingangs erwähnten Sätzen hervorgeht, keineswegs die Meinung, nur eine terminologische

*) Metamorphose $\S 3$. 
That vollbracht zu haben, sondern er sah ein Problem, dessen exakt-naturwissenschaftliche Lösung ihm freilich die Zeit, in der er forschte, schwer machen musste, das er aber für sich in seiner Weise löste durch die sich ihm aufdrängende Anschauung, dass die Blätter der "Idee" nach gleich seien. Er sagt, und dieser Satz ist der Leitsatz seiner biologischen Studien: „Dass nun das, was der Idee nach gleich ist, in der Erfahrung entweder als gleich oder als ähnlich, ja sogar als völlig ungleich und unähnlich erscheinen kann, darin besteht eigentlich das bewegliche Leben der Natur." Klarer konnte Goethe seine Anhängerschaft an Plat o's Ideenlehre nicht aussprechen.

Erst die Descendenztheorie, die den Biologen nunmehr in Fleisch und Blut übergegangen ist, vermochte eine den Naturforscher befriedigende Lösung zu bringen. Der Begriff Blatt gewann infolge dieser Theorie tieferen Ge-

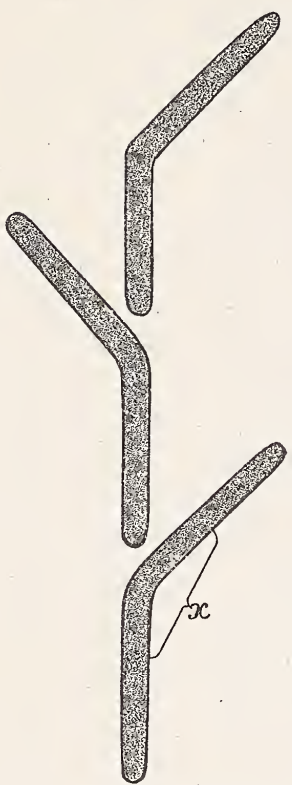

Fig. 2. Schema des Aufbaus der höh. Pflanz. nach Goethe. $x$ ist eine Einheit bestehend unten aus Stengel oben aus Blatt. halt durch die nunmehr notwendige Annahme, dass die Eigentümlichkeiten, welche so heterogene Bildungen, wie Keim-, Laub-, Kronen-, Fruchtblätter u.s. w. miteinander verbinden, sich einfach aus der Abstammung von ursprünglich gleichen Organen her erklären. Die Descendenztheorie umschliesst ja die Annahme, dass ganz allgemein kompliziertere Verhältnisse sich aus einfacheren heraus im Laufe der Generationen entwickelt haben, und so ist in unserem Spezialfall die Folgerung notwendig: die ausserordentliche Mannigfaltigkeit, welche heute die Blätter in ihrer Gestaltung und Funktion aufweisen, ist allmählich aufgetreten durch Arbeitsteilung und Uebernahme neuer Funktionen ursprünglich übereinstimmender Organe. Form und Funktion gehören ja untrennbar zusammen, sodass eine Aenderung. der letzteren mit einer Aenderung der ersteren und umgekehrt unmittelbar verknüpft ist.

Goethe hat die Einsicht, dass die Organismen in descendenztheoretischem Sinne zusammenhängen, nicht ganz gefehlt; wenigstens hat er vorübergehend diese Ansicht ausgesprochen. So sagt er: 
„Bei gewohnten Pflanzen, so wie bei anderen längst bekannten Gegenständen denken wir zuletzt gar nichts; und was ist Beschauen ohne Denken? Hier *) in dieser neu mir entgegentretenden Mannigfaltigkeit wird jener Gedanke immer lebendiger, dass man sich alle Pflanzengestalten vielleicht aus einer entwickeln könne. Hierdurch würde es allein möglich werden, Geschlechter und Arten wahrhaft zu bestimmen, welches, wie mich dünkt, bisher sehr willkürlich geschieht. Auf diesem Punkte bin ich in meiner botanischen Philosophie stecken geblieben, und ich sehe noch nicht, wie ich mich entwirren will. Die Tiefe und Breite dieses Geschäfts scheint mir völlig gleich." - Und an einer anderen Stelle: „Das Wechselvolle der Pflanzengestalten hat in mir mehr und mehr. die Vorstellung erweckt, die uns umgebenden Pflanzenformen seien nicht ursprünglich determiniert und festgestellt, ihnen sei vielmehr bei einer eigensinnigen generischen und spezifischen Hartnäckigkeit eine glückliche Mobilität und Biegsamkeit verliehen, um in so viele Bedingungen, die über den Erdkreis auf sie einwirken, sich zu fügen, hiernach bilden und umbilden zu können. Hier kommen die Verschiedenheiten des Bodens in Betracht; reichlich genährt durch Feuchte der Thäler, verkümmert durch Trockne der Höhen, geschützt vor Frost und Hitze in jedem Masse, oder beiden unausweichbar blossgestellt, kann das Geschlecht sich zur Art, die Art zur Varietät, diese wieder durch andere Bedingungen ins Unendliche sich verändern ... die allerentferntesten jedoch haben eine ausgesprochene Verwandtschaft, sie lassen sich ohne Zwang unter einander vergleichen."

Der vollen Tragweite der Annahme der Descendenz für die Probleme der Morphologie waren er, seine Zeit und die Folgezeit sich aber noch nicht bewusst. Trotzdem mussten die Thatsachen doch schon ihm und überhaupt denjenigen, die sich mit dem Gegenstande beschäftigten, Redewendungen und Worte aufdrängen, die durchaus im Sinne der Descendenztheorie liegen; aber da diese noch keinen Einfluss auf die Forschungen übte, sie aber vorläufig allein die Erscheinungen zu erklären vermag, mussten die Resultate der Morphologen einen immerhin metaphysischen Sinn gewinnen. Goethe's Ausdruck „Metamorphose“, Wendungen wie die Kronenblätter sind „umgewandelte" Staubblätter, oder wie Goethe's Bekannter, der Prof. Batsch (I795), sagt, die Anhangsorgane der Stengel ,sind nichts anderes, als mannigfaltig zur Verschiedenheit ihrer Zwecke ab g eänderte Blätter",

*) Nämlich in Italien. 
konnten damals nur bildlich verstanden werden, da eine körperliche Umänderung, Umwandlung des einen Organs in das andere, nicht beobachtet wird und der phylogenetische Begriff der Umwandlung noch nicht vorhanden war oder doch nicht berücksichtigt wurde. Dass die in Rede stehenden Autoren vermeinen, mehr als nur eine bildliche Ausdrucksweise zu gebrauchen, ist freilich richtig: man vergleiche nur die eingangs citierten Sätze Go eth e's. Es wirkt eben, wiederhole ich, hier noch die Aufsuchung von „Ideen“ im Sinne Plato's nach. Diese Auffassung kommt auch in der fleissigen Arbeit Alf. Kirchhoff's von I867 zum Durchbruch (Die Idee der Pflanzen-Metamorphose bei Wolff und Goethe p. 25), ohne dass freilich dieser Autor dabei eine Einwendung macht; denn so klar nun auch durch die Descendenztheorie der Weg für die morphologische (oder morphogenetische) Betrachtung des Blattes vorgezeichnet ist, sind doch die Einflüsse der älteren Go et he-Braun'schen Morphologie noch mannigfach auch dort übermässig zu verspüren, wo durch die Annahme der Descendenz eine vollkommene. Verschiebung der „Erklärungen“ und „Deutungen“ eintreten müsste.

Das Gefühl, dass in der Richtung, auf die die Gelekrten nachdrücklicher thatsächlich erst durch Goethe's Schrift über die Metamorphose gewiesen wurden, ein wichtiges wissenschaftliches Problem zum Verständnis der Lebewelt steckt, hat den Worten Metamorphose und Morphologie sofort Eingang verschafft. Ja in demselben Jahre, in welchem Goethe seinen Begriff Morphologie formulierte, erschien dieser Terminus schon in dem Titel einer Abhandlung, die sich ,über die Aufgabe der Morphologie" nennt und Karl Friedrich Burdach zum Verfasser hat. Die Definition, die dieser bietet, hat aus historischen Gründen Interesse; sie ergiebt sich aus den folgenden Sätzen: „Ich habe die Benennung "Morphologie" hier um so freudiger gebraucht, da Go e the vorangeht, indem er dem grossen und reichen Bilde der in seinem Gemüte sich spiegelnden Weltgestaltung jetzt die Erklärung zugesellen will, wie er die Gestaltung der Dinge in der Erkenntnis erfasst hat.“*) . . „Die Morphologie will auf dieser ihrer Höhe die Beziehung aller Gestalt zu ihrem Urquell auffassen, und den Sinn, der den Formen zum Grunde liegt, aus höherer Anschauung ableitend, eine Symbolik der Natur geben."**;)

Für eine Betrachtung der Nachfolger G o et he's ist er es selbst, der am besten weiterhilft. Das besondere Ge-

\footnotetext{
*) 1. c. p. VII.

$* *)_{0}$ l. c. p. 43 .
} 
wicht nämlich, das er gerade auf seine naturwissenschaftlichen Schriften und insbesondere auf diejenigen zur Morphologie legte, hat ihn veranlasst, eifrig die gelehrte zeitgenössische Litteratur zu studieren, um den Eindruck, den seine Lehre machen würde, zu verfolgen. Er berichtet selbst über Aeusserungen zu derselben, insbesondere über ihre Aufnahme von seiten der Botaniker. Seine Zusammenstellung "Zur Morphologie" wird von demjenigen, der an historischen Betrachtungen Geschmack findet, gern zur Hand genommen werden.

Wir ersehen aus diesen Aufzeichnungen G o e th e's, dass die von ihm in den Vordergrund gerückte Betrachtungsweise langsam aber immer breiteren Boden gewinnt, allerdings nur hinsichtlich des Vergleiches und der Beziehung der verschiedenen Blattformationen aufeinander. Es würde uns nicht fördern, alle die vielen Einzelheiten vorzuführen, sondern wir greifen - wie. das auch für die Zeit vor Goethe im Vorausgehenden geschehen ist - aus der Zeit nach Goethe und bis heute nur diejenigen Schriften heraus, die mehr oder minder weitgehende Anklänge an diejenigen Ansichten bieten, die sich dem Verfasser aufgedrängt haben. Das ist um so gebotener, als sich die meisten Autoren zunächst referierend verhalten.

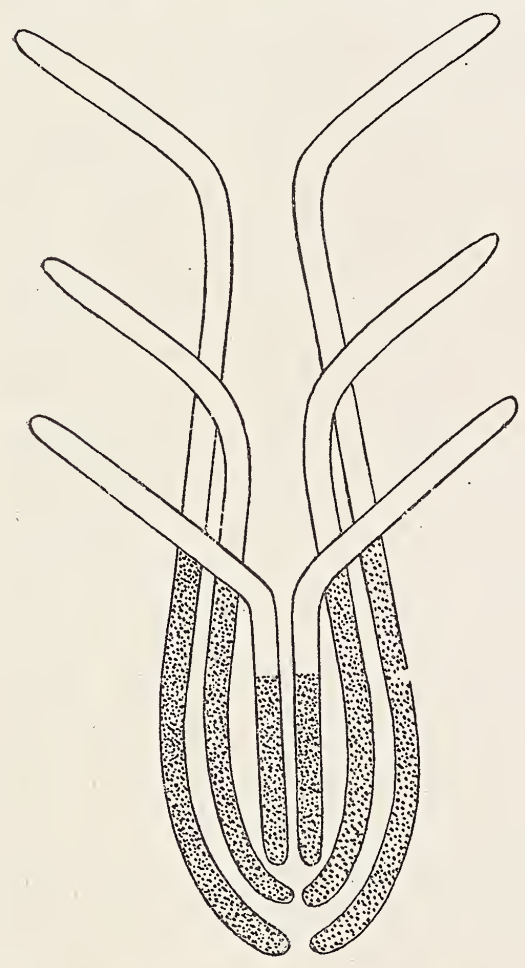

Fig. 3. Schema des Aufbaus einer höh. Pfl. nach Gaudichaud.
Hervorragend durch das Eindringen in den Gegenstand ist Ernst Meyer 1832. Er führt sämtliche Organe der Pflanze auf eines, auf das Blatt zurück: Die Blätter greifen nach oben und unten ineinander.

Gaudichaud (Recherches gén. sur l'organographie, la physiologie et l'organogénie des végétaux. Mém. de l'académie des sciences. Paris I84I) nennt das Grundorgan, aus dem die höheren Pflanzen zusammengesetzt werden, Phyton; mit seinen eigenen Worten ist ein Phyton „une feuille considérée comme plante distincte" (1. c. p. 6) oder "un végétal originel unique" (1. c. p. 38). Die 
Phyten verbinden sich in steter Wiederholung miteinander, um die höhere Gesamtpflanze darzustellen, und bestehen aus

I. einem absteigenden System (am Embryo die Wurzel),

2. einem aufsteigenden System, das in drei Teile („,mérithalles") zerfällt, nämlich
a) dem Mérithalles tigellaire,
b) " $"$ pétiolaire und
c) " " limbaire.

Figur 3 (in Anlehnung an ein von Gaudichaud selbst gebotenes Schema) giebt eine Anschauung, wie sich Gaudichaud etwa den Aufbau einer höheren Pflanze. aus Phyten vorstellte. Es sind in der Figur sechs derselben angenommen; der punktierte Teil bedeutet den absteigenden Teil, der unschraffierte den aufsteigenden Teil eines jeden Phyton.

Sehr ähnlich ist die Ansicht Hochstetter's (1847 und I848). „Blatt und darunter liegendes Halmglied (der Gräser) bilden zusammen ein Ganzes, welches er S tock werk nennt, und welches aus drei Teilen besteht, dem Fuss (Halmglied), dem Rumpf (der Blattscheide) und dem Kopf (der Blattspreite). Das nächste Stockwerk wird vom vorausgehenden erzeugt durch Abzweigung aus dem Knoten zwischen Fuss und Rumpf. In dieser Weise wird jedes Blatt als terminale Fortsetzung des Stengelgliedes betrachtet."*)

In den Jahren I843-47 taucht dann eine Lehre auf, die einen neuen Gedanken zu bringen glaubt. Es ist das die Lehre von der Anaphytose des ordentlichen Professors an der Universität zu Berlin C. H. Schultz.

Die Pflanzen, sagt Schultz**), verjüngen die einmal fertigen inneren Organe ${ }^{* * * * *)}$ nicht; sondern wiederholen den Gegensatz von Aufleben und Absterben nur in ihrer äusseren Gliederung (an den Anaphytis), indem sie über diese fertigen Gebilde hinaus immer zu neuen Produktionen, zu neuen Zweiggliedern an Wurzeln und Stengeln, neuen Blättern, neuen Knospen und Zwiebeln, neuen Knollen u. s. W. fortschreiten. Diese Eigentümlichkeit liegt in der Natur des Pflanzenwachstums, welches allein durch Wiederholung derselben Teile, durch A n a phy to sis, geschieht, während das tierische Wachstum mehr als eine Ecphys is oder Ectasis, als ein Ausdehnen der vorhandenen Teile erscheint." Schultz meint hiermit die Metamorphosenlehre zu bekämpfen, aber Alexander Braun

\footnotetext{
*) Braun, Verjüngung in der Natur I $85 \mathrm{I}$ p. I I4.

**) Die Anaphytose od. Verjüngung der Pflanzen I843.p. 89.

***; im Gegensatz zu den Tieren.
} 
macht schon mit Recht *) darauf aufmerksam, dass sich diese Lehre mit der letzteren keineswegs im Widerspruch befindet, "welche ja gleichfalls auf die ursprüngliche Gleichheit der in verschiedener Gestalt sich wiederholenden Pflanzenteile zurückführt", und Schultz selbst nennt **;) „die Ansicht, dass Wurzel, Stengel und Blätter an der Pflanze fest unterschiedene und allgemeine... Organe seien", einen Irrtum der Botanik. Die morphologische Einheit, die Schultz. nun aber als Anaphyton bezeichnet, wird von diesem keineswegs genau festgelegt.

Chronologisch wäre nunmehr A lexa nd e r Bra u n anzuschliessen; da mit ihm aber eine Richtung beginnt, die wesentlich von derjenigen abweicht, die sich ungezwungen an Goethe knüpft, setzen wir zunächst die Betrachtung der Goethe'schen Schule, wie wir in gewisser Beziehung sagen können, fort.

Durch den ausserordentlichen Einfluss, den Braun gewonnen hat, sind die Gedanken, die sich der Annahme nur eines Grundorgans mehr oder minder anlehnen, aus. dem alle übrigen Organe durch Metamorphose hervorgegangen seien, vollständig in den Hintergrund getreten, so sehr, dass sogar dahin zielende Aeusserungen aus der Feder eines Mannes wie Carl Nägeli keinerlei Wirkung. ausgeübt haben.

Dieser hervorragende Botaniker sagt einmal (I 846 p. 306), „der spätere Schein eines beblätterten Stammes rührt bloss daher, weil, wie bei jedem sprossenden Wachstum, die untersten Stücke aller successiven Achsen zusammen eine falsche Hauptachse bilden, an welcher die oberen Teile der successiven Achsen als falsche Seitenachsen befestigt sind".

Das ist im Grunde die Goethe'sche Ansicht von der Zusammensetzung der höheren Pflanzen nur aus lauter einheitlichen, gleichwertigen Stücken.

Bemerkenswert in dieser Reihe, weil mit Nachdruck und nicht bloss nebenbei betont, ist dann insbesondere die von F. Delpino geäusserte Ansicht.

Dieser Autor folgert I880 aus dem Studium der spiral gestellten Blätter, dass die Stengelorgane der höheren Pflanzen nicht in einem morphologischen Gegensatz zu den Blättern stünden, sondern ausschliesslich aus Basalstücken der letzteren gebildet seien, sodass die in Rede stehenden Pflanzen nicht "Cormophyten", sondern vielmehr "Phyllophyten" seien. Die ersten Blattanlagen verhalten sich nämlich bekanntlich wie sich berührende

*) 1. c. 1851 p. IIo.

**) I843 p. III. 
Kugeln, die diese oder jene Spiralen bilden, je nachdem sie - bei Beibehaltung der Berührung - mehr oder minder in der Horizontalen einen breiteren oder engeren Raum zur Verfügung haben, daher der Schluss D el pi n o's, dass die Stengel- (Stamm-) Organe einfach durch Verschmelzung der Blattbasen zu stande kämen.

In Frankreich glimmt der von Gaudichaud entzündete Funke noch heute schwach weiter, wie sich aus den Schriften Dangeard's ergiebt, der die „théorie de Gaudichaud", die "théorie phytonnaire" in Ehren hält. In seiner Zeitschrift „Le Botaniste" (I890-9I p. 217) spricht er z. B. von der "organisation phytonnaire" der Pflanzen, wofür die Tmesipteris-Arten ein gutes Beispiel seien und I892 (1. c.) betrachtet er die "plantules des conifères" unter dem "principe de la théorie phytonnaire".

Auch sonst ist der Begriff des "Phytons" hier und da bekannt geblieben, wie sich z. B. aus der „Anatomie et physiologie végétales" von Ed. Belzung aus dem Jahre I900 p. 334 ergiebt.

Wir kommen nun zu Alexander Braun. - Wie viel von dem, was Braun gelehrt hat, von seinem Studiengenossen und Freunde K a r $1 \mathrm{~S} \mathrm{ch}$ i m p e r beeinflusst worden ist, ist schwer zu sagen. ${ }^{*}$ ) Die eigentümliche Richtung, die als die Morphologie der Braun'schen Schule bezeichnet wird, knüpft sich nun einmal an seinen Namen, weil er es ist, der in hervorragenden Stellungen durch Wort und Schrift nachdrücklich und lange gewirkt hat. Seine Ansichten finden sich ausführlich in seiner „Verjüngung in der Natur" von 185 I dargelegt. Er ist es, der - wie gesagt - den grössten Einfluss auf die Ansichten, die die wissenschaftliche Botanik in der zweiten Hälfte des 19. Jahrhunderts in Banden gehalten haben, ausgeübt hat und noch gegenwärtig vielfach ausübt. $\mathrm{Br}$ a u n unterscheidet absolut sich gegenüberstehende Organe. Bedurften auch die Ansichten derjenigen vorausgehenden Autoren, die alle Organe auf nur eines zurückzuführen suchten, der Modifikation, und haftet ihnen auch hier und da noch viel Phantastisches an, so liegen sie doch in der Bahn der neuzeitlichen Wissenschaft und der Wissenschaft überhaupt, deren vornehmste Aufgabe in der Aufdeckung von Beziehungen, (von Zusammenhängen) besteht, für die aber Absolutes kein Gegenstand der Forschung sein kann. Insofern hat Braun einen Rückschritt gethan.

Er verwahrt sich ausdrücklich gegen eine „atomistische" Metamorphosenlehre. Der Stengel ist für $\mathrm{Br}$ a un ein

*) Vergl. z. B. G. H. Otto Volger: Leben und Leistungen des Naturforschers Karl Schimper. 3., mit erläuternden Beigaben vermehrte, Aufl. Frankfurt a. M. I889. 
"selbständiges Gebilde", „seine terminale Fortbildung erlischt ohne ein ihm selbst angehöriges Schlussgebilde". Die Wurzel hat nach Bra u n keine Metamorphose, es fehlen ihr deshalb die Blätter, ,als die Schritte im Gang der Metamorphose". "So erscheinen uns denn - sagt er p. I2O - Stengel, Blatt und Wurzel als wesentlich verschiedene Teile des vegetabilischen Organismus, als auf der Verschiedenheit der Bildungsrichtungen des Pflanzenlebens beruhende Grundorgane desselben. Ihre sichere und scharfe Unterscheidung ist die Grundfeste der Morphologie." Er betont immer wieder die „wesentliche und unwandelbare Verschiedenheit" der drei genannten Organkategorien.

Sprechen nun auch oft genug die Morphologen der Braun'schen Schule, durch den Zwang der Thatsachen veranlasst, von Uebergangsbildungen zwischen den drei Grundorganen und kommen auch oft genug Aeusserungen - auch bei Braun selbst - vor, die im Sinne der Anschauung liegen, dass alle Organe morphogenetisch zusammenhängen, so ist doch die Grundlage, von denen die Morphologen ausgegangen sind, die Annahme des absoluten Seins der drei "Grundorgane" geblieben.

Danach kann man also nur insofern von einer $G$ o e the Braun'schen Schule reden, als beide die "Ideen" zu erfassen suchten. Go e the suchte aber nur nach e in er Idee, für Braun waren die Begriffe Wurzel, Stengel, Blatt Ideen für sich. Es spezialisiert sich also die genannte Schule in zwei Richtungen, der fortgeschritteneren Goetheschen Schule und der weit rückschlägigen Braun'schen: das ist wohl festzuhalten.

Das Bedürfnis nach Zurückführung der Mannigfaltigkeit, in der die Pflanzengestaltungen erscheinen, auf Einheiten, wie das Goethe gethan hat, war aber auch bei Braun rege. Er hat als solche Einheit bei den höheren Pflanzen die Knospe bezeichnet, den Spross, den er das mit dem thierischen Individuum vergleichbare "Individuum" der Pflanze nannte (Das Individuum der Pflanze, Berlin I853). Diese Einheiten haben jedoch in der Morphologie keinerlei Rolle gespielt: ihr Aufbau bedarf ja selbst erst der morphologischen Erklärung.

Es genügte uns hier zu zeigen, dass die Ansicht Goethe's von der Zusammensetzung der. Pflanze aus einheitlichen, untereinander gleichwertigen Stücken im Gegensatz zu der Auffassung Braun's von drei sich absolut gegenüberstehenden Organkategorien, wenn auch überwuchert und vielfach verwischt und getrübt durch die Einflüsse, die er und seine Schule ausgeübt haben, doch immer wieder aufgetaucht ist und zwar ohne 
diesen Zusammenhang zu durchschauen oder einzusehen, dass Goethe's Anschauung mit den späteren - so abweichend diese auch sonst sein mögen - doch in ein und derselben Richtung liegt. Dementsprechend haben denn die Autoren ihr Resultat vielfach nicht in Anknüpfung an ihre Vorläufer, sondern vielmehr für sich durch Thatsachen erreicht, die eben immer wieder dahin drängten und zwar so stark, dass sogar ein sonst so eingefleischter Braunianer wie Č e lak o v ský (vergl. u. a. „Die Gliederung der Kaulome". Botanische Ztg. I9OI) in. Widerspruch mit der Morphologie der Braun'schen Schule doch von "Sprossgliedern" spricht, die die einzigen einen Spross zusammensetzenden morphologischen Einheiten seien.

Als ein typisches Beispiel für die Morphologie, wie sie sich in der Braun'schen Richtung gestaltet, ist das Lehrbuch der Botanik (I882) von Edmund von Freyhold zu nennen, der eine systematische Darstellung der Morphologie im Braun'schen Sinne geliefert hat.

Die Braun'sche Richtung hat lange unumschränkt geherrscht und viele wunderliche Arbeiten hervorgebracht. Geradezu beklemmend wirken die unermüdlich erfolgenden „Deutungen“ der Organe, d. h. die Erklärung eines bestimmten Organes x einmal z. B. als "Stengel“, ein andermal als „Blatt", ohne auch nur jemals ernstlicher den Gedanken zu wagen oder doch zu irgend welchen Thaten ausreifen zu lassen, dass es vielleicht Organe geben könnte, die Zwischenbildungen zwischen Stengeln und Blättern sein möchten; vielmehr erscheint durch die Vorschrift der Schule, nach der jedes Organ unbedingt einer der dogmatisch fixierten Kategorien unterzuordnen ist, das Organ $\mathrm{x}$ als ein Spielball, der nicht zur Ruhe kommt, sondern ständig und ohne Ermattung hin und her geschleudert wird: einmal in diese, ein andermal in jene Kategorie.

Dass die Begriffe Stengel, Blatt u. s. w. nicht als absolute, sondern als relative Begriffe aufzufassen sind, ist wohl gelegentlich angedeutet worden, aber die Gewohnheit hat doch die Schule im wesentlichen in der vorgezeichneten Bahn festgehalten.

Bei den ausserordentlichen Kenntnissen, die Braun auf dem Gesamtgebiet der Botanik besass, hat er - trotz der metaphysischen Grundlage, von der er ausging - die Morphologie durch eine Unzahl wichtiger Thatsachen bereichert und seine Schüler haben das Material verdienstlich gemehrt. Ein Scharfsinn besonderer Art war aber nötig, um die Thatsachen, die sich durchaus nicht der theoretischen Grundannahme der Braun'schen Morphologie fügen, doch in das Schena zu zwängen. 
Und das war und ist sogar jetzt noch möglich, trotzdem daneben die Descendenztheorie anerkannt wird, die mit der Annahme absoluter Organe in vollstem Widerspruch steht.

Braun selbst hat sogar die Descendenztheorie anerkannt (vergl. seine Rede „Ueber die Bedeutung der Morphologie" von I 862 p. $24 \mathrm{ff}$. und z. B. auch „Die Frage nach der Gymnospermie der Cycadeen" I875 p. $244 \mathrm{ff}$.) und der treffliche A. W. Eichler z. B., dessen Arbeiten sich durchaus in der Richtung der Braun'schen Morphologie bewegen, sagt ausdrücklich in seiner akademischen Antrittsrede (Berlin I880 p. 624), indem er von dieser Theorie spricht: „Der Begriff „Verwandtschaft" erlangt ... reale Bedeutung, das System wird zum Stammbaum, die Systematik zur Entstehungsgeschichte. Nichts kann wissenschaftlicher sein, als solche Forschung. Ingleichen erhebt sich die Morphologie durch Zugrundelegung der Descendenzlehre von einer schematisierenden Organbeschreibung zur lebendigen Wissenschaft von der Entstehung der Teile und ihrem genetischen Zusammenhang." Diese Erkenntnis _ - - und trotzdem auch hier die Zugrundelegung des Braun'schen Schemas in den wesentlichen Arbeiten Eichler's! Das führt so recht zum Bewusstsein, wie abhängig wir alle von Denkgewohnheiten sind, auch dann, wenn wir bei besonderer. Ueberlegung die Hinfälligkeit ganz. wichtiger unter ihnen erkennen.

Nur spät und sehr allmählich versuchten es Einzelne, die Botanik durch energischen Protest aus diesem Bann zu erlösen, der die Wissenschaft zur Metaphysik der aristotelisch-platonischen Schule(!), also in das graue Altertum zurückzuschleudern auf dem Wege war. Von diesen Protestlern ist in erster Linie Julius Sachs $\mathrm{zu}$ nennen.

Julius Sachs und sein Schüler Karl Goebel sind bemüht, die Organgestaltung und somit den Gesamtaufbau der Pflanzen überhaupt aus den Lebenserscheinungen derselben heraus zu begreifen, wenigstens neigt in den letzten Stadien ihre Meinung dahin, dass nach voller Aufdeckung der Funktionen auch die Gestaltungen erklärt seien.

$\mathrm{Sachs}$ hat seine diesbezüglichen Ansichten besonders durch seinen Aufsatz über Stoff und Form (I880) eingeleitet, mit dem er ausdrücklich die Morphologie der Braunschen Schule bekämpft. In der materiellen Beschaffenheit der Organe, im Zusammenwirken mit den Einflüssen ihrer Umgebung sieht er die Ursachen der organischen Formen. Veränderungen der organischen Formen beruhen auf den Ernährungsvorgängen. Die spezifisch organbildenden Stoffe werden durch äussere Einflüsse, speziell durch de Schwere und das Licht, in der Art affiziert, dass da- 
durch in gewissen Fällen die räumliche Anordnung verschiedener Organe bestimmt wird. So werden unter dem Einfluss intensiven Lichts gewisse Bildungsstoffe in den Laubblättern erzeugt, die spezifisch zur Blütenbildung geeignet sind. Man wird annehmen müssen, dass es zur Bildung der Stoffe zu den Laubblättern, den Blüten u. s. w. nur der Anregung durch gewisse minimal vorhandene Stoffe (Enzym e) bedarf, aber nicht, dass der gesam te nötige Stoff schon in den Stengeln etc. vorhanden sei.

Es ist ganz zweifellos, dass diese Forschungsrichtung höchst wertvoll ist. Doch ist damit das Problem der Morphologie noch nicht erschöpft und umgrenzt und mit der Aufdeckung der Beziehungen zwischen Stoff und Form ist der Werdegang der höheren, komplizierteren Pflanzen aus einfacheren, die Art der phylogenetischen Aneinanderknüpfung der Organismen noch nicht eruiert. Diese Beziehungen gestatten wohl Einsichten nach der Richtung, woher es komme, dass unter Umständen, die das Experiment schaffen kann, z. B. aus einer Anlage, die nach üblichem Verhalten ein Laubblatt geworden wäre, ein Sporophyll entsteht, jedoch bleiben nun noch die wichtigen Fragen übrig, wie sich die Organe morphogenetisch aneinanderknüpfen, wie und aus welchen primitiveren Organen man sich z. B. die Entstehung der Laubblätter, der Sporophylle, der Blütenblätter u. s. w. zu denken hat.

Goebel*) will in erster Linie die organographischen Thatsachen aufsuchen, die sich durch Funktionen erklären : er will die Beziehungen aufdecken, die zwischen Funktion und Form vorhanden sind, eine Forschungsrichtung, die sehr fruchtbar ist und die daher vielfach gepflegt wird, so insbesondere von $\mathrm{Sch}$ wend e ner und seiner Schule. Go ebel beschäftigt sich vorwiegend mit der Aufdeckung dieser Beziehungen, soweit sie die Gesamtorgane (die Organe höherer Ordnung) betreffen, die uns bei äusserer Betrachtung der Pflanzen entgegentreten, Schw endener's Schule, soweit sie sich in der histologischen Struktur zu erkennen geben.

Die Forschungsrichtung der Morphologie hingegen sucht die Entstehung der Organe aus Ur-Anfängen zu begreifen, und das ist zu erreichen durch Studium der Beziehungen, die die Organe der Vorfahren mit denen der Nachkommen verknüpfen.

Es sind noch viele andere Forschungsrichtungen möglich: eben so viele wie Beziehungen denkbar sind. Diese Richtungen schliessen einander natürlich nicht aus, sondern sie ergänzen sich: erst alle Beziehungen, die I898-I 901 .

*) Vergl. insbesondere seine „Organographie der Pflanzen". Jena 
zwischen Pflanzen und Anderem möglich sind, sofern sie zu höheren Begriffen führen, bilden die Wissenschaft der Botanik. Unter "Richtung" ist demnach in diesem Sinne weiter nichts $\mathrm{zu}$ verstehen als die besondere Pflege einer durch praktische oder sonstige Gründe gepflegten Gruppe von Thatsachen und Beziehungen; nicht aber ist gemeint, dass die verschiedenen Forschungsrichtungen unvereinbare Gegensätze schaffen, wie man etwa jetzt noch in der Philosophie seine Untersuchungen von einer bestimmten Richtung aus anstellt in dem Sinne, dass hier die Thatsachen von.den verschiedensten Seiten benutzt werden, um unvereinbare Systeme zu stützen. Vielmehr liegen die Forschungsrichtungen, die hier gemeint sind, alle in derselben Bahn: in derjenigen der naturwissenschaftlichen Methodik, die darauf hinausgeht, alle Beziehungen im Einzelnen aufzudecken und dadurch $\mathrm{zu}$ höheren Begriffen fortschreitend $\mathrm{zu}$ einer aus diesem sich ergebenden einheitlichen Ansicht über die Pflanze, _ _ - schliesslich (bei Mitberücksichtigung aller übrigen naturwissenschaftlichen Disziplinen) zu einer Weltansicht zu gelangen.

Diese Andeutung war notwendig, um nicht den Gedanken aufkommen zu lassen, als handele es sich in der Morphologie in dem von mir dargestellten Sinne etwa um eine Disziplin, die aus dem Rahmen des bisherigen naturwissenschaftlichen Betriebes fällt, die den Anspruch macht, fundamental Verschiedenes von dem $\mathrm{zu}$ bieten, was sonst die Naturforschung leistet, als wolle sie gar die Grundlagen der letzteren umformen. Nein: sie ist einfach ein notwendiges Glied im Ganzen, ohne welches dieses kein Ganzes sein würde.

In einer Hinsicht ist die Morphologie gegenüber der organographischen Richtung im wesentlichen Nachteil. Bei der Aufsuchung der Anknüpfung der Organe an solche, die bei den Vorfahren vorhanden gewesen sind, fehlt ihr der Grad der Sicherheit, der bei organographischen Studien vorhanden ist, dass heisst, es fehlt meist die Möglichkeit direkter experimenteller Prüfungen. Sie ist vielmehr u. a. darauf angewiesen, die so lückenhafte Kenntnis, die wir über die ausgestorbenen Pflanzen besitzen, zu Hilfe zu ziehen; es ist ihr nur untergeordnet möglich Experimente zu verwerten, da sich das Wichtigste, die Phylogenesis der Organismen, nicht wiederholen lässt. Es muss daher der Morphologie obesonders viel Theoretisches anhaften; und so sind denn Fehler hier leichter $\mathrm{zu}$ begehen als in irgend einer anderen Disziplin. Das darf uns nun aber nicht abhalten, den Versuch zu machen, hier so weit zu kommen, als es eben möglich ist, in der Hoffnung, mit der Zeit und durch viele Mitarbeit eine Ausgestaltung dieser. 
wichtigen Disziplin zu erreichen, die allseitige Anerkennung zu finden vermag.

I 898 und I899 habe ich denn nun durch Gelegenheitsschriften, und wo sich sonst Veranlassung bot*), meine Auffassung über die Morphologie der Pflanzen in nuce kundgegeben, und ich habe die Absicht, dieselbe in einem besonderen Werk eingehender, als es bisher geschehen konnte, zu begründen.

Kurz und bündig würde das Resultat, das ich gewonnen habe, sich wie folgt präzisieren lassen.

Die Gestaltungen der Organismen sind ein Produkt aus ihrer materiellen Zusammensetzung und den Einflüssen der Aussenwelt, kurz: die Bestimmungsgründe ihrer Formen sind ,innere" und ,äussere". Gelangen sie in andere Verhältnisse oder ändert sich ihre Umgebung, so suchen sich die Organismen derselben anzupassen, wodurch sie sich - falls sie die Anpassung erreichen - umgestalten: wir haben dann Anpassungs-Charaktere. Die verschiedensten Organismen können sich gleichen Verhältnissen anpassen, also die gleichen Anpassungs-Charaktere erlangen, die aber aus ihrer abweichenden Herkunft erklärliche Verschiedenheiten beibehalten. Je länger nämlich solche Anpassungs-Charaktere, d. h. in ihrer Form mit ihrer Funktion harmonierende Eigentümlichkeiten in einer Reihe von Generationen bestanden haben, um so schwieriger wird es bei eventuellen Neuanpassungen sie wieder $\mathrm{zu}$ beseitigen oder zu modeln, und wenn nunmehr Formänderungen eintreten, so kann das wieder nur im Anschluss an das Gegebene und durch Benutzung desselben geschehen. Diese Ansicht möchte man trivial nennen und doch wird diese wichtige Thatsache nicht in dem Masse berücksichtigt, wie es nötig ist. Das vor der Ummodelung gegeben Gewesene wird sich nachher mehr oder minder deutlich erkennen lassen und diese dauernderen Eigentümlichkeiten, die sich unter Umständen nicht in voller Harmonie $\mathrm{zu}$ der Neuanpassung befinden, sind die morphologischen Charaktere (Organisationsmerkmale Nägeli's [Abstammungslehre I884 p. 327]).

Die letztgenannten Charaktere sind also ursprünglich ebenfalls Anpassungscharaktere gewesen.**)

*) Vergl. insbesondere I) Lehrbuch der Pflanzenpalaeontologie. Berlin 1897-1899. 2) Die Metamorphose der Pflanzen im Lichte palaeontologischer Thatsachen. Berlin $1898 . \quad 3)$ Die morphologische Herkunft des pflanzlichen Blattes und der Blattarten. Berlin r899. 4) Abstammungslehre und Darwinismus. Berlin (wie die vorigen Ferd. Dümmler's Verlagsbuchhandlung) I899.

**) Auf den oben angedeuteten Zusammenhang zwischen Anpassungsund morphologischen Charakteren habe ich zuerst in meiner Schrift: Die Abstammungslehre, Berlin 1899, S. I18-124, aufmerksam gemacht. 
Es ist klar, dass sie es sind, deren Studium die echte Verwandtschaft der Lebewesen untereinander $\mathrm{zu}$ erkennen ermöglicht, während die Neuanpassungen höchstens dadurch verwirren, als eben Lebewesen der abweichendsten Herkunft unter Umständen durch nachträgliche Anpassung an gleiche Verhältnisse auch in gewissen Punkten genau dieselben Eigentümlichkeiten gewinnen können.

So wird man nicht schliessen, dass etwa der Walfisch, weil er fischähnliche Flossen besitzt, nun auch mit den Fischen zunächst blutsverwandt sei.

Es ist immer zu unterscheiden zwischen alten, älteren, neuen und neuesten Anpassungen, um bezüglich der Erkennung der Blutsverwandtschaft zu richtigen Resultaten zu gelangen: stets sollte sich der Morphologe Rechenschaft über das Alter der Umbildung von Organen, über das Alter ihm entgegentretender Anpassungserscheinungen zu geben suchen.

Bei der Wichtigkeit des aufgestellten Gesetzes, welches die vergleichsweise Beständigkeit der morphologischen Charaktere gegenüber den Anpassungscharakteren zum Ausdruck bringt, wollen wir dasselbe noch einmal mit anderen Worten wiederholen.

Die Umbildung eines Organes $a$ in ein Organ $b$ begegnet um somehr inneren, d. h. im Lebewesen liegenden Hindernissen, je weiter in den Generationsreihen (d.h. phylogenetisch) die Zeitzurückliegt, in der das Organ $\alpha$ entstanden. war. Morphologische Charaktere sind bei den Vorfahren Anpassungs-Charaktere gewesen.

Wenn also Pflanzen, deren gesamte Blätter noch den beiden Hauptverrichtungen - in deren Dienst überhaupt alle sonstigen Lebenserscheinungen stehen - , nämlich der Ernährung und der Fortpflanzung, dienen (welche Trophosporophylle sind), eine Arbeitsteilung dadurch einleiten, dass die Blätter sich bei den Nachkommen in zwei Sorten scheiden und dementsprechend nur noch der Ernährung oder nur noch der Fortpflanzung dienen, wie das bei gewissen Farnen vorkommt, die diese beiden Blattsorten (Trophophylle neben Sporophyllen) entwickeln, so ist die Möglichkeit, durch geschickte Eingriffe aus Anlagen, die Sporophylle erzeugt hätten, nun reine Trophophylle zu erhalten, grösser, als etwa solche Anlagen zu bewegen, Trophosporosome, d. h. der Ernährung und Fortpflanzung dienende Thallusstuicke, zu werden. In der That kann man, wie Goebel gezeigt hat, durch gewisse Eingriffe jene Blattsorten gelegentlich ineinander verwandeln. 
Als Gegenstück zu diesem Beispiel sei ein anderes erwähnt. Wenn man nach Vöchting*) den Kohlrabi (Brassica oleracea f.gongylodes) am Blühen verhindert und alle Achselknospen vernichtet, schwellen die Blattkissen knollig an und füllen sich mit Reservestoffen (besonders mit Calciumphosphat), wodurch also Ablagerungsstellen für Substanzen entstehen, die sonst in den Samen abgelagert worden wären. Diese „samenbildenden“ Substanzen haben aber in diesem Falle doch keine Samen gebildet!

Ein wichtiger Beweis für unser Gesetz sind ferner die Thatsachen, die man bei Kreuzungen erzielt.

Verschiedene Arten, Rassen oder Varietäten, z. B. Pferd und Esel, oder aber die verschiedensten Hunderassen untereinander können sich miteinander geschlechtlich vermischen ( $\mathrm{sich} \mathrm{kreuzen}, \mathrm{bastardieren)}$ Dem Tierzüchter ist von seinen Rassen vielfach bekannt, wann sie entstanden sind; nehmen wir nun einmal eine Rasse $A$ und eine andere $B$, und wissen wir, dass die Rasse $A$.sehr viel länger besteht als die Rasse $B$, so können wir voraussagen, dass die Mischlinge aus beiden in ihrem Aeussern und Innern mehr nach $A$ hin neigen werden als nach $B$. Bei Kreuzungen wirken die älteren Formen stärker als die neueren; nur dann entsteht eine genaue Mittelform zwischen beiden Eltern, wenn die Formen, von denen beide abstammen, phylogenetisch gleich alt sind. Die morphologischen Merkmale eben die ältesten und älteren Merkmale - besitzen eine grössere Vererbungskraft als die neueren Anpassungscharaktere, die sich nur der Zeit ihrer Entstehung nach, aber sonst in keiner prinzipiellen Weise von den morphologischen Merkmalen, die - wiederhole ich - ursprünglich auch Anpassungscharaktere waren, unterscheiden. ${ }^{* *}$ )

Auffällige und zahlreiche Thatsachen haben mich nun zu der Annahme geführt:

Die Blätter der höheren Pflanzen sind im LaufederGenerationenaus Thallusstücken wie Fucus gegabelter Algen oder doch algenähnlicher Pflanzen hervorgegangen, dadurch dass

*) Zur experimentellen Anatomie. - Nachrichten der K. Ges. d. Wiss. zu Göttingen. Mathem:-physikal. Klasse 1902.

**) Ich freue mich, dass Herr Prof. v. Wettstein (Neubildungen von Formen im Pflanzenreich. Ber. d. deutsch. bot. Ges. I90o p. [194]) diesen Gedanken acceptiert hat, wenn auch vorläufig erst in der folgenden Fassung. Mit Bezugnahme auf meine diesbezügl. Aeusserung p. I I 8 meiner Abstammungslehre sagt er: „Ich denke an die Möglichkeit der allmählichen Umwandlung von Anpassungsmerkmalen in Organisationsmerkmale. Diese Möglichkeit ist entschieden vorhanden und würde zugleich erklären, warum zahlreiche Organisationsmerkmale ausgesprochen zweckmässige Einrichtungen sind." 
Gabeläste übergipfelt und die nunmehrigen Seitenzweige $z u \cdot B l a ̈ t t e r n$ (im weiteren Sinne, zunächst zu Ur-Blättern) wurden. Die übergipfelnden Stücke werden zu Achsen (Ur-Caulomen, Centralen).

Die Achsen der niederen Pflanzen (Algen) unterscheiden sich von denen der höheren dadurch, dass an dem morphologischen Aufbau der letzteren die Blattbasen teilnehmen. Es ergiebt sich die Notwendigkeit, die Stengel und Stämme der höheren Pflanzen als in ihrer morphologischen Natur zusammengesetzt anzusehen. Damit würde sich das Blatt, wie es uns bei gewissen Algen, z. B. bei Sargassum entgegentritt, von dem Blatt der höheren Pflanzen unterscheiden, indem das erstere seine Grenze an der Ansatzstelle desselben an der Achse findet, während das morphologische „Blatt" der höheren Pflanzen an der Stengel- und Stammbildung teilnimmt.

Nur zwei wesentliche Stücke: I. die Centrale (das Ur-Caulom) und 2. das Ur-Blatt sind es, die durch Umbildung im Verlaufe der Generationen die Gesamtheit aller Formgestaltungen der höheren Pflanzenwelt bedingen; da diese beiden Stücke phylogenetisch aus Gabelästen von Thalluspflanzen sich herleiten lassen, s o is t s c hli es slich das eine und einzige morphologische Grundorgan aller höheren Pflanzen ein thal1 öses Gabelglied.

Schematisch veranschaulicht wird diese Theorie durch die Abbildungen der Figur 4.

Zum Verständnis dieser Theorie von dem morphologischen Aufbau der höheren und höchsten Pflanzen ist daran festzuhalten, dass mir in erster Linie daran gelegen ist, den Versuch zu machen, aufGrund der bisherigen Thatsachen der gesamten Botanik (auch derPalaeobotanik) das phylogenetische Werden der Pflanzenformen, die phylogene. tische Entstehung des Aufbaus der höheren Pflanzen zu verstehen.

Eine Bekämpfung der Theorie bloss durch entwicklungsgeschichtliche Thatsachen ist nicht angängig; es bleibt daher nur übrig, in erster Linie die fertigen $\mathrm{Zu}$ stände der fossilen und rezenten Pflanzen in ihrer formalen Aneinanderknüpfung in Rücksicht zu ziehen, denn die entwicklungsgeschichtlichen Thatsachen dürfen zwar einer morphologischen Theorie nicht widersprechen, da Ontogenie und Phylogenie zusammengehören, allein man darf von der Entwicklungsgeschichte (der Ontogenie) nicht erwarten, dass sie morphologische Theorien positiv und unbedingt stützen $\mathrm{m}$ ü s s e. Es hiesse das verlangen, das jedes Individuum in seiner Entwicklung ab ovo all und jede 


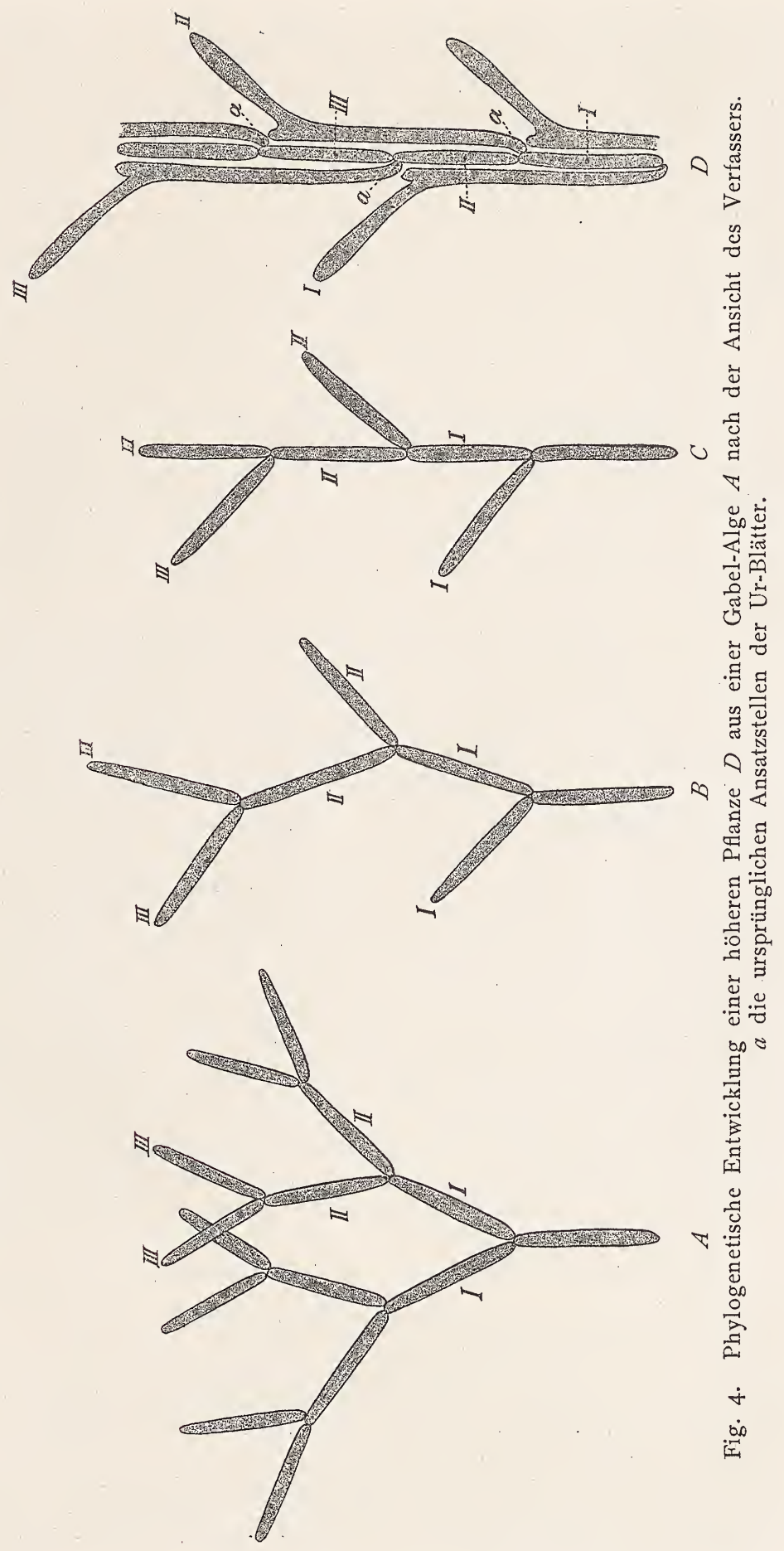


Eigentümlichkeiten sämtlicher Vorfahren wiederhole, während die Thatsachen dafür sprechen, und es auch viel näher liegt, anzunehmen, dass die Organismen in ihrer individuellen Entwicklung möglichst schnell, also auf dem kürzesten Wege ihren fertigen Zustand zu erreichen trachten. Ernst Mehnert*) z. B. weist sogar darauf hin, dass functionell hochstehende Organe sehr häufig früh, of t v o r i h re n phyletischen Mutt erorgan en angelegt werden, dass sich dementsprechend atavistische Bildungen spät entfalten.**) Er kommt zu dem Schluss: Die individuelle Entwicklung ist nicht eine nur verkürzte, sondern eine im einzelnen verschobene Wiederholung der phyletischen; die Abweichungen werden durch die functionelle Höhe oder Bethätigungsstärke des Organs beherrscht. In der individuellen Entfaltung ist die Ausbildung jedes Einzelorgans ein relativ selbständiger Vorgang. Entwicklung oder Rückbildung tritt ein durch Vererbung der im individuellen Leben erworbenen Kräftigung oder Verkümmerung des Organs.

Es ist daher von vornherein ein verkehrtes Beginnen, von der Entwicklungsgeschichte definitive Antworten auf phylogenetische Fragen zu beanspruchen. Eine Entscheidung, ob im Verlaufe der Generationen die Basalstücke, deren Gipfel die Blätter der höheren Pflanzen sind, mit dem Träger (der Achse) zu einem einheitlichen Gebilde verwachsen sind, vermag die Entwicklungsgeschichte am Individuum nicht $\mathrm{zu}$ liefern.

Die Farne und Pteridophyten überhaupt weisen darauf hin, dass sie von echt-dichotom-verzweigten Vorfahren abstammen ${ }^{* * * *}$ ); gehen wir möglichst weit zurück, so werden wir daher kaum fehlgehen - da man doch Algen oder algenähnliche Pflanzen an den Anfang setzen muss - in einem Aufbau, wie ihn etwa Fucus zeigt, einen Urtypus $\mathrm{zu}$ erblicken, von dem die Phylogenese der höchsten Pflanzen ausgegangen ist $\dagger$ ). In Fig. $4 \mathrm{~A}$ ist der Typus schematisch zur Darstellung gebracht und die drei daneben abgebildeten Schemata B, C und D sollen kurz veranschaulichen, wie ich mir den Uebergang von diesem Bau-

\footnotetext{
*) Biomechanik, erschlossen aus dem Prinzip der Organogenese. Jena 1898 .

**) So wird das Herz eher als Arterien und Venen angelegt, diese werden eher als die Blutkörperchen gebildet etc.

***) Vergl. meine Abhandlung: Die Beziehung zwischen dem echtgabeligen und dem fiederigen Wedelaufbau der Farne (diese Berichte Bd. XIII, I895, S. $244 \mathrm{ff}$.) und mein Lehrbuch der Pflanzenpalaeontologie, Lief. I, Berlin I897, S. $16 \mathrm{ff}$.

$\dagger)$ Ich halte es für richtiger homolog zu setzen: den Algenkörper mit der embryonalen Generation der Pteridophyten. Näheres in meinem Lehrbuch der Pflanzenpalaeontologie, Berlin, 2. Lief., I897, S. I59.
} 
typus zu demjenigen der höchsten Pflanzen (D) vorstelle. Durch Uebergipfelungen von Gabelzweigen erhalten wir durch die Zwischenform B Pflanzen C (wie z. B. die Sargassum-Form), die sich bereits in Achsen (Centralen) und Anhangsorgane gliedern, und durch streckenweises Verwachsen der Basalstücke der letzteren mit den Achsen erhalten wir (wie D veranschaulicht) den Aufbau der höchsten Pflanzen aus Stengeln (Centrale + den Basalstücken der Anhangsorgane) und Blättern (das sind die freibleibenden Enden der Anhangsorgane).

Ich bezeichne übersichtlich als

Trophosporosome Körper resp. Organe (also in A die einzelnen Gabelstücke) von Thallophyten, die sowohl der Ernährung als auch der Fortpflanzung dienen, als

Trophosome solche, die nur oder wesentlich der Ernährung dienen, als

Sporosome solche, die nur oder wesentlich der Fortpflanzung dienen, als

Ur-Caulom (Centrale), die durch Uebergipfelung von Gabelzweigen, die zu Anhangsorganen werden, hervorgehende Achse (vergleiche $\mathrm{C}$ ); letztere wird zum Träger. Die Anhangsorgane (Ur-Blätter) sind entweder

Ur-Trophosporophylle (Ante-Trophosporophylle), d. h. Ur-Blätter (Ante-Phyllome), die sowohl der Ernährung als auch der Fortpflanzung dienen, oder

Ur - Trophophylle (Ante-Trophophylle), d.h. Ur-Blätter, die nur oder wesentlich der Ernährung dienen, und

Ur-Sporophylle (Ante-Sporophylle), d. h. Ur-Blätter, die nur oder wesentlich der Fortpflanzung dienen.

Bei den höchsten Pflanzen sind zu unterscheiden ausser der Centralen (dem Ur-Caulom) derjenige Stengeltheil, der diese Centrale wie einen Mantel umgiebt und aus den Basalstücken der Ur-Blätter hervorgegangen ist, nämlich das

Pericaulom und ferner die Anhangsorgane der Stengel (die freigebliebenen Enden der ursprünglichen UrBlätter), das sind die Blätter im gewöhnlichen Sinne (= Post-Phyllome, Caulom-Blätter), die sich scheiden in

(Post-) Trophosporophylle,

"Trophophylle (Laubblätter) und die

" Sporophylle u. s. w.

Als Modell - um zu illustrieren was ich meine mag die Achse eines noch geschlossenen Pinus-Zapfens einmal das Ur-Caulom vorstellen, die Apophysen würden den Blattpolstern entsprechen und die miteinander verwachsen zu denkenden Fruchtschuppen selbst dem Pericaulom. Bei der Ananas, wo der gesamte Fruchtstand - 
dessen Achse Deckblätter trägt, in deren Winkel die Früchte stehen - eine einheitliche Masse bildet, kommt diese ja durch eine vollständige Verwachsung der Deckblätter und Früchte miteinander thatsächlich zu Stande. Bei einem wirklichen Pericaulum sind allerdings die miteinander verwachsenden Basalteile der Anhangsorgane meist sämtlich steiler nach aufwärts gerichtet zu denken.

Aus der vorn gebotenen historischen Betrachtung geht hervor, dass schon mancherlei an die Pericaulum-Theorie Anklingendes geäussert worden ist, geleitet durch die Thatsachen, die immer wieder darauf hinweisen werden, dass der "Stengel" der höheren Pflanzen in morphologischer Beziehung kein einheitliches Gebilde ist. Es ist nur durch die Ueberwucherung, durch die Braun'sche Morphologie verständlich, dass sogar dahin zielende Aeusserungen aus der Feder eines Meisters der Forschung, wie W. Hofmeister, keinerlei. Wirkung ausgeübt haben.

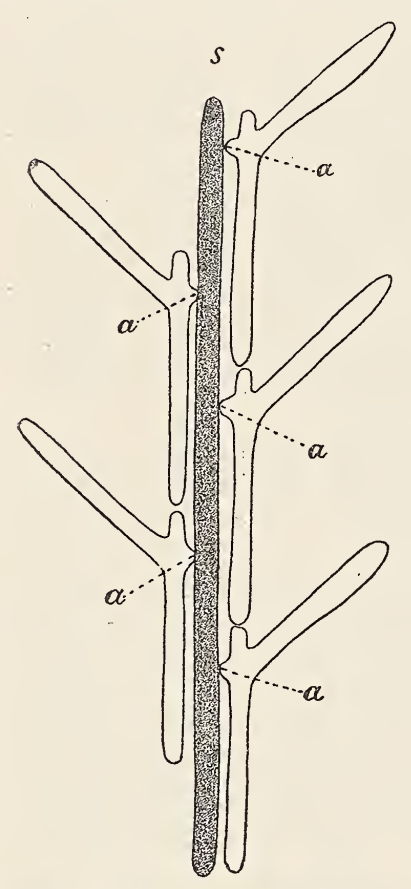

Fig. 5. Schema des Aufbaus einer höheren Pflanze nach der Ansicht $\mathrm{H}$ of $\mathrm{m}$ ei is te r's. $s=$ Stengel, $a=$ ursprüngliche Ansatzstellen d. Blätter.

Hofmeister sagt (vergl. Untersuch. I 85 I, S. 90) bezüglich der Entwicklung von Equisetum. „Die starke Vermehrung des unteren Teiles der äusseren Blattfläche wandelt bald die Blattbasis zu zahlreichen, der Längsachse des Sprosses parallelen, de $n$ äusseren Umfang des Stengels darstellenden*) Zellschichten um. Auf der Vermehrung dieser, aus der Entwicklung des Basilarteils der Blattanlage hervorgegangenen Zellenmasse in die Länge und Breite beruht a u s schliesslich*) die fernere Längen- und Dickenzunahme der Stengelglieder. Der unmittelbar aus der Endknospe hervorgegangene centrale Zellcylinder des Stengels wird ganz und gar zum Marke."

Für diese Ansicht (vergl. das Schema Figur 5) von der Berindung derStengelorgane durch Wachstum der Blattbasen, hat $\mathrm{H}$ of $\mathrm{m}$ e is $\mathrm{ter}$ noch wiederholt eine Lanze gebrochen. So sagt er über

*) Die hier gesperrt gedruckten Worte sind auch bei Hofmeis ter hervorgehoben gedruckt! $-\mathrm{P}$. 
zehn Jahr später (in einer Besprechung einer Arbeit Stenzel's in der „Flora“ von I863, S. I73): „Die Entwicklungsgeschichte des Stengels der Farn stellt es für ihn, wie für die unendliche Mehrzahl der bisher untersuchten beblätterten Pflanzèn von den Charen aufwärts ausser Zweifel, dass eine Berindung des Stengels von den Basen der jüngsten Blätter aus erfolge. Die Interfoliarstücke entwickelter Stengel, mögen sie auch nach späterer Vermehrung und Dehnung ihrer Zellen in Richtung der Länge noch so sehr gestreckt sein, noch so scharf von den Blättern gesondert erscheinen - sie bestehen gleichwohl bei der grossen Masse der beblätterten Pflanzen in ihren äusseren Schichten aus Geweben, die ursprünglich Teile der dicht gedrängten Blätter waren." Wiederum später, in seiner „Allgemeinen Morphologie“" (Leipzig I 868, S. 520) hat sich $\mathrm{H}$ ofm ei ster über den Gegenstand abermals geäussert. „Die Berindung des Stengels durch das Dickenwachstum der Basen der jungen Blätter — sagt er hier - ist ein überaus weit verbreiteter Vorgang." Auch hier geht er wiederum von Chara aus, um verständlich zu machen, was er meint. - Das Schema Fig. 5 wird im Vergleich zu den im Vorausgehenden gebotenen Schematen auf den Unterschied seiner Anschauung von denen der anderen Autoren und namentlich von der Pericaulom-Theorie schneller hinweisen, als es Worte thun können. a sind die Ansatzstellen der Blätter (weiss gelassen) an den "Stengel“ (dunkel gehalten).

Ebenso wie diese höchst beachtenswerten Winke Hofmeister's haben sich die Bemerkungen zum Gegenstande eines Mannes wie Carl Nägeli fast verloren, obwohl sich nicht nur aus dem Anfange seiner wissenschaftlichen Laufbahn (vergl. vorn S: I6), sondern auch in seinem "wissenschaftlichen Vermächtnis" von I 884 wichtige Aeusserungen finden.

$\mathrm{N}$ äg eli sagt (Abstammungslehre I884, Anmerkung): „Der unterste Teil eines Blattes ist in dem Gewebe des Stengels eingesenkt, wie sich sehr deutlich an den verkümmerten Blättern von Psilotum zeigt." Er erklärt ferner ganz allgemein (S. 457), dass das Basalgewebe des Blattes mit dem Caulom verschmolzen sei. Und ferner lesen wir bei diesem Autor (S. 477-78): „Wenn auch die Sporogonien bei den meisten Selaginellen nicht wie bei Lycopodium an der Blattbasis, sondern dicht über den Blättern aus dem Stengel zu entspringen scheinen, so muss ich sie doch für blattständig halten, denn ein Teil des morphologischen Blattes (im Gegensatz zum äusserlich erkennbaren) ist jedenfalls in das Gewebe des Stengels eingesetzt .... Das die sichtbare Blattbasis zunächst umgebende 
Gewebe der Stengeloberfläche gehört also höchst wahrscheinlich dem Blatte an".

Auch Graf zu Solms-Laubach sei erwähnt, der in seiner Palaeophytologie (I887, S. 200 und 248) vom Lepidodendron-Blattpolster sagt: „Das ganze Polster entspricht dem am Stamm verbliebenen, herablaufenden Blattgrund." Und über die längsrippigen Stammoberfächen der Rhytidolepen (einer Sigillaria-Gruppe) macht er die Bemerkung: "Jede Längsrippe des Rhytidolepis-Stammes kommt durch Verschmelzung der senkrecht übereinander stehenden Blattpolster zu Stande."

Ich betone ausdrücklich, dass ich auf die Anschauungen der Autoren, die namentlich hinsichtlich des Vorhandenseins eines Pericauloms anklingende oder mehr oder minder hinneigende Gedanken geäussert haben, erst dann geachtet und zum Teil überhaupt erst kennen gelernt habe, nachdem die Thatsachen mich selbständig zu der PericaulomTheorie geführt hatten. Erst das Bedürfnis in der Litteratur, nach eventuellen Vorgängern oder nach Anklängen zu fahnden, die mich zu fördern vermöchten, auch die Neigung dem Historischen gerecht zu werden, hat mich mit den anklingenden Aeusserungen in der Litteratur bekannt gemacht. Es geschieht dieser Hinweis, um den Eindruck zu verstärken, dass die Theorie wohl der eingehenderen Berücksichtigung wert ist, da eine ganze Anzahl Autoren, und unter ihnen Männer wie H of meister und Näg eli, von ganz verschiedenen Seiten aus in mehr oder minder nahe sich berührenden prinzipiellen Punkten in die gleiche Bahn getrieben worden sind. Ich hätte noch eine ganze Anzahl Autoren dafür citieren können, wie Spring (I849), Leitgeb (I868), Fle ischer (I874), Goebel (I883 und später), De Vries, L. J. Čelakovský u. a.

Uebersichtlich ergeben sich die Unterschiede in den Auffassungen der Morphologie der Stengelorgane wie folgt:

I. Der Stengel ist ein Organ für sich und steht in vollem Gegensatz zu den Blättern (z. B. Wolff und A. Braun).

II. Der Stengel hat Blatt- resp. „Phyton“- Natur, er wird ausschliesslich von den Basalteilen von Blättern gebildet (Goethe, Gaudichaud).

III. Der Stengel hat in seinem Centrum Achsennatur, in seiner Peripherie Blattnatur.

I. Die Achse wird durch das Auswachsen der Basis der Blätter berindet (z. B. Hof m e is te r).

2. Die Achse, das Urcaulom, erhält durch ihre im Verlaufe der Generationen stattfindende Verwachsung mit den Basalteilen ihrer blattförmigen 
Anhänge (Urblätter) einen Mantel: ein Pericaulom. Das letztere entsteht durch Zusammenaufwachsen der Basalteile der Urblätter.

Manche Aeusserungen der Autoren lassen sich nicht in eine dieser Kategorien bringen, da sie oft zu allgemein gehalten sind. Meinungen dahin gehend, dass die Stengeloberflächen Blattnatur hätten, finden sich mehrfach, ohne dass jedoch nähere Andeutungen geboten würden, aus denen sich ein Gedanke über die phylogenetische Entstehung dieser Blattnatur ergäbe. Legen wir uns die Ansicht Hofmeister's phylogenetisch zurecht, so müssten wir die Pflanzen, deren Stengel er durch Blattbasen berindet annimmt, aus solchen hervorgegangen denken, die wie bei Chara (die ja auch Hofmeister als Vergleich heranzieht) in ihrer individuellen Entwicklung noch deutlich eine nachträgliche Berindung aus den Basalzellen der Anhangsorgane aufweisen, während meiner Vorstellung nach die Basalstücke der Anhangsorgane selbst eine ganze Strecke mit den Achsen verwuchsen (mit diesen zusammen aufwuchsen) und so ein Pericaulom gebildet haben. Von einem Auswachsen aus der Basis der Anhangsorgane zur Berindung der Achsen ist also in der letzterwähnten Theorie im Gegensatz zur Hofmeister'schen nicht die Rede (vergl. die schematischen Figuren 4, $D$ und 5). Auch nehmen nach der Pericaulom-Theorie die Blattbasen in sehr viel weitergehendem Masse an der Zusammensetzung der Stengelorgane teil, als dies meist die Autoren zugeben, die der Berindungshypothese zuneigen, nach der nur die äusserste Rinde (die Blattpolster und dergl.) ihre Herkunft aus den Blattbasen erkennen lassen.

Ich gehe nun auf die Thatsachen zur Begründung der Pericaulom-Theorie ein.

I. Die Ontogenese giebt hier wenig Winke. Man kann nicht erwarten, dass der Vorgang der PericaulomBildung, der - ihre Richtigkeit vorausgesetzt - in der geologischen Formation stattgefunden haben müsste, als die ersten Landpflanzen entstanden, jetzt noch an den höchsten Pflanzen entwicklungsgeschichtlich rekapituliert werde. Die Thatsache, dass die Keimpflanzen der Fïlices nur ein centrales Bündel in ihren Stengelchen besitzen (die also monostel sind), auch dann, wenn die erwachsenen Pflanzen in ihren Stengelorganen z. B. einen Kreis von Bündeln aufweisen (also polystel sind), deutet jedoch wohl darauf hin, dass erst später eine Vereinigung der Blattfüsse zu polystelen Stengeln stattgefunden hat.

Die Entwicklung der Keimpflanzen der höheren Pflanzen ist, soweit sie für unsere Frage in Betracht kommt, hin- 
reichend in Kny's Arbeit „Die Entwicklung der Parkeriaceen" (Nova acta der Ksl. Leop.-Carol. Deutschen Akademie der Naturforscher. Dresden I 875, S. 62 ff.) resumiert. Er macht darauf aufmerksam, dass die Untersuchungen am Embryo durch Hanstein, Hieronymus, Fleischer und $\mathrm{Hegelm}$ aier darin übereinstimmen, „dass der vordere Teil der Keimanlage zum Cotyledo wird und die Knospe seitlich in einer an seinem Grunde sich bildenden Vertiefung entsteht. Innerhalb der Knospe wird nicht, wie man erwarten sollte, sofort der Stammscheitel konstituiert, welcher seinerseits den Blättern den Ursprung zu geben hätte, sondern es wird der am Grunde des Cotyledo sich emporwölbende Höcker zum ersten Laubblatt, aus dessen Basis geht dann in ähnlicher Weise das zweite Laubblatt hervor, bis erst bei allmählichem Erstarken der Keimpflanze die Stammspitze sich selbständig gestaltet." Danach müsse man mit Fleischer, sagt Kny weiter, die Keimpflanze als ein Sympodium indifferenter Sprossungen (Thallome) betrachten, von denen jedes sich erst später in Stamm und Blatt zerlegt. Nach seiner eigenen Untersuchung gewähre es auch bei den Keimpflanzen der Farn den Anschein, „als ginge jeder Wedel aus der Basis des nächst älteren hervor". Selbst Nägeli sage, dass es unmöglich sei, zwischen Stamm und Blatt zu unterscheiden, und Kny citiert hierzu des ersteren Satz: „Der spätere Schein eines beblätterten Stammes rührt bloss daher, weil, wie bei jedem sprossenden Wachstum, die untersten Stücke aller successiven Achsen zusammen eine falsche Hauptachse bilden, an welcher die oberen Teile der successiven Achsen als falsche Seitenachsen befestigt sind." Kny fügt nun aber seinerseits hinzu: „Auch bei den Keimpflänzchen von Ceratopteris stimmte der erste Eindruck, welchen ich empfing, mit der Auffassung von Nägeli durchaus überein. Doch liess sich der Nachweis führen, dass ein selbständiger Stammscheitel an der Knospe des Farnembryo wirklich besteht und dass er nur von den sich unter ihm entwickelnden Wedeln rasch überholt und zur Seite gedrängt wird."

Sehen wir uns Kny's Figuren auf Taf. IV (XXI), $\mathrm{V}$ (XXII) und VI (XXIII) an, so bemerken wir in der That eine führende Zelle, die nun schon als Andeutung des „Stammes" im Gegensatz zu den Blättern angesehen wird. Will man aber auf Grund einer solchen Zelle eine schroffe Heterogenität zwischen einem sonst nicht sichtbaren Organ, dem „Stengelorgan“ und Seitenorganen, den „Wedeln" oder „Blättern" postulieren, so muss dasselbe überall da geschehen, wo solche führenden Zellen vorhanden sind, also z. B. auch beim Farnwedel der ebenfalls 
eine Scheitelzelle erkennen lässt. Der Wedel müsste dann ebenfalls als aus zwei morphologisch heterogenen Elementen aufgebaut angenommen werden und überhaupt alle Organe, die eine Scheitelzelle unterscheiden lassen.

Ich muss daher im Rahmen meiner Theorie die Sache so ansehen *), dass bei den Farn die „Stamm"scheitelzelle homolog dem nächsten darunter angelegten Wedel ist, der jedoch in Anknüpfung an die Herkunft der höheren Pflanzen von Gabelalgen, die durch Uebergipfelungen dichopodiale Sympodien bilden, nun noch immer in seiner embryonanalen Entwicklung an dieses phylogenetische Werden durch Uebergipfelung der "Scheitelzelle" erinnert. Dass bei späteren, d. h. noch höheren Pflanzen diese Erinnerung endlich ganz ausgelöscht ist, wie z. B. bei Elodea mit dem äusserst charakteristischen "Stammscheitel“, an dem von Uebergipfelungen nichts mehr wahrzunehmen ist, ist durchaus begreiflich, d. h. steht durchaus in Zusammer.klang mit der Annahme der allmählichen Auslöschung von Entwicklungsverhältnissen, die für den fertigen Zustand keine Bedeutung mehr haben, und es ist gleichzeitig festzuhalten, dass auch neue, den fertigen Zustand schneller und besser errreichende Momente in die Erscheinung treten können, die sich dann nicht mehr an Früheres anknüpfen lassen.

2. Es ist bemerkenswert, dass schnell aufwachsende Sprosse (man kann ja durch Schnitt im Sommer Pflanzen veranlassen, besonders schnell wachsende Sprosse zu bilden) das sogenannte "Herablaufen“ der Blätter sehr viel auffälliger zeigen, als langsamer erwachsene; es ist zu beachten, dass ganz allgemein schnelleres Wachstum atavistische Erscheinungen bedingt. ${ }^{* *}$ ) Dabei ist es natürlich für die Entscheidung der Frage nach der Berechtigung der Pericaulomtheorie ganz gleichgültig, ob diese „herablaufenden" Teile sich später als die Blattprimordien entwickeln, ob also wirklich in diesem bildlichen Sinne ein Herablaufen stattfindet oder nicht. Die spätere Entstehung von herablaufenden Flügeln an Stengeln von Cirsium, Symphytum u. s. w. kann höchstens für diese Theorie ausgenutzt werden, insofern als die Laubblätter der höheren Pflanzen vorwiegend am Gipfel zuerst fertig sind und die Basalteile erst später den fertigen Zustand erreichen. Eichler giebt***) an, dass sich der Blattstiel nach der

*) Es ist wohl zu beachten, dass auch die Meinung, die in Rede stehende Scheitelzelle sei ein Hinweis auf das Vorhandensein eines zu den Blättern gegensätzlichen Stammes durchaus nur eine Deutung ist, die ebenfalls bloss im Rahmen einer bestimmten Theorie nöglich ist.

$* * *$ ) Vergl. meine Abstammungslehre (Berlin I899, S. I IO).

***) Zur Entwicklungsgeschichte des Blattes. Marburg 186I, S. 9. 
Spitzenanlage entwickle; es würde danach sehr auffällig sein, wenn die "herablaufenden" Blattteile sich zuerst entwickeln würden. Das Scheitelwachstum dauert bei den Blättern der Angiospermen nur ganz kurze Zeit*), meist ist es schon $\mathrm{zu}$ Ende, wenn die Blattanlage noch nicht einmal einen Millimeter Länge erreicht hat; die Längenzunahme erfolgt dann also intercalar und vielfach z. B. basifugal; das stimmt ganz mit dem späteren Auftreten von herablaufenden Flügeln, das also in der That wohl für aber nicht gegen die Pericaulomtheorie spricht.

Es sei daran erinnert, dass auch bei Fucaceen und anderen Algengruppen intercalares Wachstum eine ganz allgemeine Erscheinung ist.

3. Sodann sei erwähnt, dass die palaeozoischen Lepidophyten (die Lepidodendren, Bothrodendraceen und die Sigillariaceen), sei es ihr ganzes Leben hindurch, sei es nur in ihrer Jugend resp. an ihren dünneren (jüngeren) Zweigen, sich mit wohl abgegrenzten Blattpolstern derartig besetzt zeigen, dass zwischen diesen weiter keine Oberfläche am Stamm übrig bleibt. Ein voll entwickeltes Lepidodendron-Polster zeigt die Blattnarbe, darüber eine Ligulargrube und unter der Narbe zwei lenticellenartige Transpirationsöffnungen, ähnlich denen auf den Blattfüssen mancher tropischer Farne. Die den Stamm bekleidenden Blattpolster sind aber Basalstücke der Blätter und bilden die Peripherie des Stammes. Bei den Bothrodendraceen mit ihren kleinen, weit voneinander stehenden Blattnarben, muss man aus der Thatsache, dass auch sie in der Jugend wie die Lepidodendren gepolstert sind und die Polstergrenzen sich nach Massgabe des Dickenwachstums auslöschen, schliessen, dass die Stammflächen zwischen den Blattnarben ebenfalls aus den Basalstücken der Blätter hervorgegangen sind. Auch an den dicksten Stammresten der Bothrodendraceen ist das übrigens noch dadurch erweisbar, als sich oberhalb der Blattnarben (also auf der Stengelfläche zwischen den Narben) die Ligulargruben deutlich markiren. Ueber die Sigillariaceen endlich ist das Folgende zu sagen: Es muss angenommen werden, dass die Vorfahren der polsterlosen Sigillariaceen oder diese in ihrer Jugend Blattpolster besessen haben, so dass die freilich an Stammoberflächen stärkerer Reste meist nicht abzugrenzende Umgebung der Blattnarben als zum Blatte (als dessen Basis) gehörig anzusehen ist. $\mathrm{Zu}$ einer Blattbasis würde oberhalb der Blattnarbe der Teil zu

*) Vergl. Paul Sonntag, Ueber die Dauer des Scheitelwachstums und Entwicklungsgeschichte des Blattes. Inaugural-Dissertation. Berlin I 886. 
rechnen sein, der die Ligulargrube trägt, und unterhalb der Narbe der Teil, der die eigentümlichen lenticellenartigen Oeffnungen, die „Transpirationsöffnungen“ trägt.*) Einen Sinn hat die Zurechnung der Blattnarbenumgebung zum Blatt nur durch die Annahme, dass bei der Stammbildung der Pflanzen im Verlaufe der Generationen die untersten Teile der Blattstiele, resp. Blattteile nach und nach vollkommen mit dem ursprünglichen Stamm, der Centrale, verwachsen sind. Diese Annahme wird durch die Reihenfolge des Auftretens der Sigillarien sehr schön unterstützt: in der chronologisch 4. Flora sind namentlich die gepolsterten Sigillarien zu Hause, während die Sigillarien der 5. und 6. Flora, vorwiegend die Blattnarben auf Rippen stehen, die durch Längsfurchen zu Stande kommen, ohne dass jedoch die Narben oben und unten von den nächststehenden durch Querfurchen geschieden werden, und endlich gelangen wir zu den Sigillarien der 7. Flora bis zu der Pleuromoia des Buntsandsteins, bei denen die Polsterabgrenzung vollständig verlöscht ist oder doch nur nebenbei vorkommt.**)

Bei den rhytidolepen Sigillarien ist die Abgrenzung der als solche nicht individuell abgehobenen, sondern das Pericaulom bildenden Blattfüsse noch oft dadurch markiert, dass sich dicht über die Ligulargrube eine mehr oder minder gerade oder bogenförmige Abgrenzungslinie befindet, die bei Ablehnung der Pericaulomtheorie ebenso wenig erklärlich sein würde, wie die die Narbenorthostichen trennenden Längsfurchen. $\mathrm{Zu}$ einem das Pericaulom mit bildenden basalen Blattteil würde aber hier, soweit die an der epidermalen Oberfläche des Stammes sichtbaren Teile in Betracht kommen, gehören: zunächst die Blattnarbe, sodann der die Ligulargrube besitzende Flächenteil über derselben bis zu der erwähnten queren Begrenzungsfurche und

*) Letztere sind allerdings erst bei einer einzigen Sigillaria (vergl. mein Lehrbuch der Pfianzenpalaeontologie. Berlin I899, S. 245, Fig. 230) bekannt geworden.

**) Ich habe mich in Obigem absichtlich möglichst wörtlich an meine früheren Darstellungen des Sachverhalts (vergl. „Die Metamorphose der Pflanzen im Lichte palaeontologischer Thatsachen", Berlin 1898, S. 20 ff. und Lehrbuch der Pflanzenpalaeontologie, Berlin I899, S. 245) gehalten, um recht auffällig zeigen zu können, wie sehr diese Darstellungen missverstanden worden sind. Friedrich Tobler sagt nämlich in seiner vorn citierten Arbeit: „Die Funde an Sigillariaceen, vom Carbon bis zum Buntsandstein, sollen alle Uebergänge darbieten von kleinen Blattbasen bis zu solchen, die den ganzen Stamm bedecken und ihn demnach berinden." Das soll eine Wiedergabe meiner Auseinandersetzung über den thatsächlichen Sachverhalt sein! Ich kenne keine Sigillarien mit kleineren Blattbasen, die den Stamm noch nicht ganz „,berinden"; vielmehr sind alle Sigillariaceen bereits hochorganisierte Pericaulom-Pflanzen. 
drittens die unter der Narbe vorhandene Fläche bis zur Querfurche oberhalb der nächstunteren Narbe. In Uebereinstimmung mit dem unter 2 über das intercalare resp. basiläre Wachstum der Blätter Gesagte

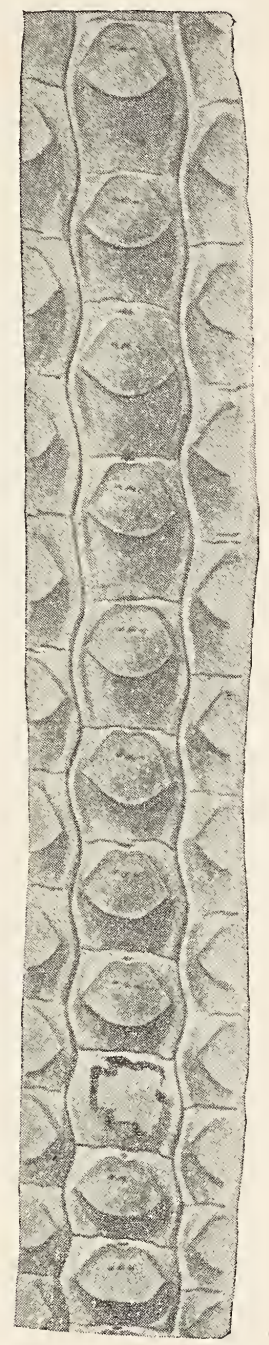

Fig. 6.

Sigillarie, die durch Querfurchen noch die Polstergrenzen zu erkennen giebt. zeigt sich nun bei diesen Sigillarien und den favularischen Stammoberflächen, dass der oberhalb der Blattnarben befindliche Teil bis zur Querfurche gegenüber der unter der Blattnarbe vorhandenen Fläche an dem Längenwachstum des Stammes garnicht oder kaum teilnimmt; vielmehr ist es nur der ligulargrubenfreie, untere Teil, der sich in die Länge streckt, wie das unsere Figur 6 auffallend $\mathrm{zu}$ erkennen giebt. Diese Erscheinung bliebe ohne die Annahme des Vorhandenseins eines Pericauloms ganz unklar, während sie bei der Voraussetzung eines solchen in den Rahmen der übrigen Erscheinungen passt, hier insbesondere der allgemeineren Thatsache sich unterordnet, dass das Längenwachstum des Blattes vorwiegend und am ausgiebigsten an ihrer Basis erfolgt. Die erwähnte Eigentümlichkeit bei den Sigillarien erklärt sich also leicht durch die phylogenetische Entstehung des peripherischen Stengelteils aus verwachsenen (Ur-)Blattbasen.

Auch die Lepidodendren zeigen übrigens den Uebergang von der Polsterung zur Leiodermie gelegentlich sehr schön und dies sogar bei ein und derselben Species. Mir sind Arten aus dem Culm (Lepidodendron Volkmannianum) und aus dem produktiven Carbon bekannt, die diese Erscheinung zeigen. Lepidodendron Volkmannianum ist gewöhnlich gepolstert und zwar können (r.) die Polster ringsum ohne Unterbrechung geschlossen sein; (2.) oft jedoch zeigen sich Unterbrechungen oben und unten, wodurch ein Anklang an die Rhytidolepis-Skulptur zuwege kommt, wie er auch sonst gelegentlich bei Lepidodendron beobachtet wird; (3.) bei der in Rede stehenden Art können endlich auch die seitlichen Polstergrenzen schwinden und wir haben dann eine epidermale Oberfläche, die im 
Quincunx und entfernt voneinander Blattnarben trägt, ohne dass diese irgendwie von Polstergrenzen umgeben werden. Was Graf Solms über Lepidophyten und Nägeli über Selaginella sagt, wurde schon S. 31/32 erwähnt.

Für den, der übrigens noch weitere Thatsachen wünscht zur Erweisung, dass die gesamten Stammoberflächen höherer Pflanzen aus Phyllombasen hervorgegangen sind, sei noch auf das Verhalten der seinerzeit auch in diesen Berichten*) besprochenen Lepidodendraceengattung Lepidophloios hingewiesen. Bei dieser Gattung bleiben am Stamm nach dem Blattfall Blattfüsse stehen, und zwar schuppenförmige Gebilde, die auch der energischste Gegner der Pericaulomtheorie, sofern er auf dem Boden der üblichen Botanik steht, ebensowenig in morphologischer Hinsicht zum Stengelorgan rechnen würde, wie man die bei Farn oft stehenbleibenden Basalteile der Wedelstiele (ebenfalls also von Blattfüssen) jemals anders als eben als Basalteile von Wedeln angesehen hat.

Die (nach abwärts gerichteten) șchuppenförmigen Blattfüsse von Lepidophloios tragen auf ihrer oberen Fläche die Ligulargrube, auf ihrer unteren die Transpirationsöffnungen. Im Vergleich mit der eng verwandten Gattung Lepidodendron wären demnach hier nur zwei Möglichkeiten offen, nämlich entweder gehört darnach das Lepidodendron-Blattpolster ebenfalls zum Blatt oder aber die Blattfüsse von Lepidophloios sind merkwürdige Auswüchse des Stammes, auf deren Gipfeln die Blätter sitzen. Dann aber müsste man doch auch die Blattfüsse der Farn morphologisch zu den Stammorganen rechnen: das ist mit Recht bis jetzt niemals geschehen.

4. Man kann übrigens die Pericaulomnatur der Stengel der höheren Pflanzen auch auf ihre anatomischen Verhältnisse begründen. Vor allem ist da an die Thatsache zu erinnern, dass die allermeisten Pflanzen nur Blattspuren in ihren Stengeln aufweisen, während "stammeigene“ Bündel selten sind. Nun wird man erwidern, dass doch die Blätter notwendig in ihrem Leitungssystem eines Anschlusses an das gemeinsame System in den Stengeln bedürfen und damit stehe notwendig das Vorhandensein von Blattspuren in Verbindung. Ich müsste dem insofern widersprechen, als damit die Auffälligkeit unerklärt bliebe, warum denn nun die Blattspuren, um diesen Anschluss zu gewinnen, nicht den bequemsten direkten Weg wählen, sondern erst eine ganze Strecke abwärts durch die Rinde verlaufen. (Man denke z. B. an die Stengelanatomie von Lycopodium u. s. w.) Der Hanstein'sche Terminus

*) „Die Zugehörigkeit von Halonia". Bd. XI, 1893, S. 484 ff. 
„Blattspur" (Ueber den Zusammenhang der Blattstellung mit dem Bau des dicotylen Holzringes. Jahresber. über die städt. Gewerbschule, Berlin I857) ist daher sehr treffend und unter dem Eindruck der auffälligen Einheitlichkeit der Blattspuren als den unteren Stücken von Blättern verliehen. Hanstein's Fig. Io ist diesbezüglich charakteristisch. Wäre der „Stengel“ der höheren Pflanzen gegenüber den „Blättern“ ein so fundamental gegensätzliches Organ, so hätte der genannte Autor nach sorgfältigen Untersuchungen nicht zu dem Satze gelangen können, „dass der dicotyle Holzring seine erste Entstehung einer Anzahl von gesonderten Gefässbündeln verdankt, welche ganz und unmittelbar in die Blätter übergehen, und ausser welchen keine primären Bündel existieren." Die Blätter bestimmen nach seiner Darlegung - und dies ist ja alles jetzt jedem Botaniker ganz geläufig - die Gestaltung des Holzringes, und wir können hier sagen, diejenige des Stengels. Kurz der Stengel der höchsten Pflanzen zeigt sich vom Holzring inklusive bis zur Epidermis entwicklungsgeschichtlich-anatomisch nicht aus zweierlei Organen, sondern aus nur einheitlichen Stücken zusammengesetzt: den Blättern und ihren miteinander verschmolzenen (zusammen aufwachsenden) Basalteilen.

Bei Farn ist die Zugehörigkeit der einzelnen Stengelbündel zu den Wedeln noch besonders auffällig auch im fertigen Zustande zu eruiren.

5. Danach kann also in den Stengeln der höchsten Pflanzen nur etwa der Markkörper der Centrale, der Urachse, entsprechen. Da diese ursprünglich - bei ihrer Gleichwertigkeit mit den Ur-Trophosporophyllen (resp. UrTrophophyllen oder -Sporophyllen) - ebenfalls in ihrem Centrum leitende Elemente besassen, kann es nicht Wunder nehmen, dass wir. gelegentlich auch bei den höchsten Pflanzen mit sonst bündellosem Markkörper doch gelegentlich, sei's als atavistische Erscheinung**), sei's regelmässig (z. B. bei Begonia, Melastomaceen) markständige Bündel vorfinden. Wiederum sind es die Pteridophyten, die treffliche Uebergänge zeigen.

Hydroidenzwischenformen zu Markzellen sind bei Isoëtes, Lepidodendron und Megaloxylon vorhanden. Bei Medullosa anglica und anderen Arten ist an stelle des „Markes“ ein vollständiges Primärxylem entwickelt.

Vergleichen wir die unter einander verwandten Sphenophyllaceen, Protocalamariaeeen und Calamariaceen, so ist der Schluss, dass die Leitbündelelemente im Centrum

*) Vergl. Th. Künkel, Strangbildungen im Marke von Alnus glutinosa. Botan. Centralblatt. Bd. 72, Cassel I897. 
im Verlaufe der Generationen verschwinden, um dieselben nur im Pericaulom übrig zu lassen, sehr gut begründbar. Vergleiche diesbezüglich das, was ich u. a. in den Natürlichen Pflanzenfamilien I, 4, S. 56I-562 gesagt habe. Um hier ein anderes Beispiel anzuführen, sei erwähnt, dass die beiden verwandten Gattungen Heterangium, die vom Culm ab vorkommt, und Lyginopteris, die erst vom unteren produktiven Carbon ab bekannt ist, dadurch unterschieden sind, dass Heterangium gewöhnlich eine dünnere Lage von sekundärem Holz besitzt und ein gut entwickeltes Centralbündel, während bei der erst später auftretenden Form Lyginopteris an Stelle der Central-Stele ein Markkörper vorhanden ist und ein starker sekundärer Holzring.

Bei den Cycadaceen, die ebenfalls ältere Typen sind, sind isolierte Leitbündel im Marke als normale Erscheinungen bekannt, so bei Encephalartos. Im Mark von Cephalotaxus*) sind Tracheiden vorhanden.

Wir haben also alle Uebergänge von centralen Leitbündeln auch dort, wo die Bündel im Umkreise der Pflanze genügen würden, bis zum abnormen, gelegentlichen Vorkommen von Bündelelementen in einem sonst wohl ausgebildeten Mark. Der Markkörper der Stengelorgane oder ein Teil derselben würde also morphologisch dem Ur-Centralbündel entsprechen; bei Isoetes z. B. können somit die Mark-Spiralzellen als letzte Rudimente des ursprünglichen centralen Stammleitbündels, das bei den Vorfahren vorhanden war, gelten. In dem Markkörper der höheren Pflanzen dürften wir somit das morphologische Aequivalent des Ur-Cauloms zu erblicken haben.

Von Wichtigkeit ist es fur die Frage nach dem Vorkommen von markständigen Leitbündeln, dass dort, wo es sich um Ausnahmserscheinungen wie bei Almus handelt, solche Bündel sich vorwiegend in den schneller aufwachsenden Trieben $\mathrm{zu}$ finden scheinen ( $\mathrm{K}$ ü nkel 1 . c. p. I, 2 des Separatabdrucks). Freilich muss das noch eingehender geprüft werden, da $\mathrm{K}$ ünkel die Abnormität überhaupt nur in 3 Trieben (darunter 2 Schösslinge) konstatiert hat. Ich selbst habe sie in dem mir gerade zur Verfügung stehenden Material nicht finden können. Schneller aufwachsende Organe - wiederhole ich - sind für Rückschläge geeigneter als langsam wachsende (vergl. S. 35).

Dass unter den Pflanzen mit stammeigenem Bündel die Wasserpflanzen einen hervorragenden Platz einnehmen, ist besonders bemerkenswert. Bedeutet doch die Anpassung höherer Pflanzen an das Wasserleben ein Zurückgehen in Verhältnisse, die für weit weit entlegene Vor-

*) Vergl. Rothert, Ber. d. Deutsch. botan. Ges., I899, S. 275 ff. 
fahren die üblichen waren, womit auch im Bau ein Zurückgehen in alte Zustände bedingt ist.

6. Auch dem anatomischen Bau der Leitbündel selbst sind Daten zu entnehmen, die darauf hinweisen, dass dieselben als ursprünglich konzentrisch gebaut anzunehmen sind und erst im Verlaufe der Generationen zu collateralen geworden sind. Darauf weist in erster Linie das häufige Vorkommen diploxyler Bündel im Palaeozoicum hin, wie solche bei jetzt noch lebenden älteren Typen wie den Cycadaceen partiell erhalten sind.

7. Gewisse Thatsachen über Gewebespannungen sind vielleicht ebenfalls auf die Pericaulom-Bildung zurückzuführen. Ërnst $K$ üster hat nachgewiesen ${ }^{*}$ ), dass bei allen von ihm untersuchten Algen (Florideen und Fucaceen u. a.) die Gewebespannung sich insofern gerade umgekehrt verhält wie sie in den Stengelorganen der höheren Pflanzen konstatiert werden kann, als bei den ersteren die äusseren Gewebeschichten, die „Rindenschichten“, sich bei einer Trennung von den centralen verlängern, also die "Markschichten" sich entsprechend verkürzen. Bei den höheren Pflanzen jedoch verkürzen sich bekanntlich im allgemeinen losgetrennte Rindenstücke, während ein Stück des Markkörpers sich verlängert.

Nach der Pericaulom-Theorie, nach der der Markkörper der höheren Pflanzen morphologisch etwa den übergipfelnden Trophosomen, die Peripherie des Stengels jedoch und die Blätter als Fortsetzungen den übergipfelten Trophosomen entsprechen würde, ist es danach begreiflich, wenn letztere in der Energie des Längenwachstums von den ersteren übertroffen werden, nach der Regel nämlich, dass die Organe, die sich der Senkrechten am meisten nähern, auch diese Energie am stärksten zeigen. Das Pericaulom muss daher von der Centralen, deren Streben nach Längenwachstum intensiver ist, mitgenommen werden.

8. Dass Abnormitäten sich in Zusammenklang mit einer guten morphologischen Theorie bringen lassen müssen, ist für mich zweifellos. Sie sind ebenso Naturerscheinungen wie alles Andere. Die neuerliche Neigung sie beiseite zu schieben und möglichst unbeachtet zu lassen, ist ein Ausdruck für die Unbequemlichkeit, die sie den gegenwärtigen, nicht haltbaren morphologischen Theorien schaffen: der Prüfstein für eine brauchbare Theorie ist die Möglichkeit alle Formgestaltungen durch Zuhilfenahme derselben erklären d. h. mit einander in Beziehung setzen zu können.

Ich wähle als Beispiel das altbekannte von der Birne. Die Fähigkeit der Birnenfrucht gelegentlich ihr äusseres

*) Sitzungsber. der Akad. des Wiss. in Berlin, I899. 
Fruchtfleisch in richtige Schuppen aufzulösen, die sogar noch in grüne spreitige Teile ausgehen können, weist auf die Phyllomnatur des Fruchtfleisches hin. Man könnte hier ganz gewiss im Sinne von $\mathrm{Sachs}$ sagen, dass wohl besondere Ernährungs- und sonstige äussere Verhältnisse vorgelegen haben, die die Entstehung von Blättern auf der Birne zur Folge hatten und in der That ist es auch so. So blühten z. B. nach Gustav Cab a ǹ s (Bull. Soc. d'étude sciences nat. Nîmes I897/98) infolge des milden Herbstes I 897 viele Obstbäume in der Nähe von Nîmes zum zweiten Mal, und manche trugen auch noch einmal Früchte. Diesen sassen aber grüne Laubblätter auf, auch mitten auf dem Fruchtfleisch, die kleiner als sonst Birnblätter, im übrigen aber vollkommen ausgebildet waren. Der Grund für die Deformation liegt nun darin, dass die zweiten Früchte sich nicht unter den üblichen Bedingungen entwickeln konnten; es fehlte ihnen die nötige Temperatur, Nahrung u. s. w. Dies ist die Ansicht des genannten Autors.

Gewiss: auch ich zweifle nicht im mindesten daran - und schöne Experimente von Peyritsch, Sachs und Goebel beweisen dies -, dass bei der Entstehung von Abnormitäten äussere und Ernährungsverhältnisse mitspielen. Die Aeusserungen, die an den Pflanzen durch diese Einflüsse zuwege kommen, liegen aber in der Bahn des Gesamtaufbaues des ergriffenen Pflanzenteiles. In unserem Falle bleibt aber die Frage übrig, warum, wenn gerade "Laubblattstoff" erzeugt wird, dieser so und immer in der bestimmten Weise zur Verwendung kommt, dass beim Vorhandensein von Birnenfruchtanlagen das Fleisch derselben sich $\mathrm{zu}$ Schuppen und Laubblättern gestaltet, und die Antwort kann keine andere sein als die: weil die Nahrung nur solche Bahnen:leicht beschreiten kann, die gewohnte sind oder doch gewesen sind, weil Neubildungen auf Grund dieser spezifischen Nahrung nur da entstehen werden, wo die nächste Anknüpfung an diese neuen Organe zu finden ist. Wenn also das Fruchtfleisch der Birne morphologisch im Verlaufe der Generationen aus verwachsenen Blattbasen hervorgegangen ist, so liegt es am nächsten, hier für den durch abnorme Verhältnisse gebildeten "Laubblattstoff" einen Ausweg zu finden durch Bildung von Blättern gerade an Stelle des Birnenfleisches.

So einfach, wie das nun aber oben zur Darstellung gelangt ist, also aus Centrale und einfachem Pericaulom, sind die Stengel der höheren Pflanzen nicht zusammengesetzt, vielmehr ist zu. erkennen, dass die Neigung zur Pericaulombildung dauernd bestanden hat. Wir werden 
also annehmen, dass eine Pericaulompflanze durch $\mathrm{Zu}-$ sammenaufwachsen ihrer Stengel mit den basalen Teilen ihrer Blätter unter Umständen ein sekundäres Pericaulom bilden konnte u. s. w.

Dann ist aber noch zum Verständnis der Mannigfaltigkeit der Stengelbildungen zu beachten, dass nicht nur Centralen und Pericaulome mit ihren Appendices (Blättern u. dergl.) zusammen aufwachsen können, sondern auch Stengelorgane untereinander. Darauf weisen die Schlingpflanzen hin. Bei dem an Etiolement erinnernden schnellen Längenwachstum der Lianenstengel, die aus dem Dunkeln ans Licht streben, bleiben dieselben vergleichsweise dünn; durch Zusammenaufwachsen mehrerer derselben wird die nötige Stärke erreicht und zugleich die für solche Pflanzen zweckmässige Taukonstruktion,

In dem Abschnitt "Cycadofilices" habe ich in den "Natürlichen Pflanzenfamilien" I, 4, S. 78I-782 ff. (I90I) auseinandergesetzt, inwiefern man für die Cycadales kletternde Filicales als Vorfahren vermuten könnte. Ihr „anormales" Dickenwachstum deutet u. a. darauf hin; diese sogenannten Anomalien sind ja für Lianen charakteristisch. Das Vorkommen von Peridermlamellen im Stamme bei gewissen recenten Cycadaceen, die bis ins Mark hineingreifen, und dieselbe Erscheinung bei mesozoischen Bennettitaceen, in beiden Fällen also wiederum bei älteren Typen, würde ich als Hinweis auf die Zusammensetzung der Stämme aus verwachsenen Stengelorganen ansehen, die aussen ein Periderm besassen. Bei Stangeria entstanden Knospen an Stücken von Querscheiben, die in Erde gesetzt worden waren, a uf der Markseite des Holzkörpers.*) Diese Thatsache bleibt - wie so viele - für die Braun'sche und gegenwärtige Morphologie gänzlich unerklärbar, d. h. lässt sich nicht in Beziehung zu bereits Bekanntem setzen. Für die Pericaulomtheorie liegt in dieser Thatsache jedoch gar nichts Ueberraschendes, da die Entstehungsstelle dieser ,abnormen“ Knospen der ursprünglichen Aussenfläche eines (nunmehr verwachsenen) Stengelorganes entspricht.

Ueber die Veranlassung zur Bildung von Pericaulomen habe ich mich schon früher geäussert (vergl. Die Metamorphose der Pflanzen im Lichte palaeontologischer Thatsachen, Berlin I898, S. 25-27). Das Resultat lautet dort: Ein Pericaulom entsteht durch das Bedürfnis, einen festen Hohlcylinder für die aufrechten Stämme der zum Luft-

*) Vergl. Renault, Cours de botanique fossile, I88I, I, p. 4I, der diese Beobachtung Houllet's mitteilt. 
leben gekommenen Pflanzen $\mathrm{zu}$ haben; das wird eben im Anschluss an das Gegebene am besten durch Verwachsung zunächst der Trophosom- resp. Trophosporosom-Basen erreicht. Da aber dann die letzteren die Leitung der Nahrung in Richtung der Stammlänge besorgen, wird das ursprüngliche Centralbündel überflüssig, dessen schliessliches Verschwinden überdies dadurch unterstützt werden muss, als die mechanische Konstruktion im Centrum der festen Elemente, die bei den meisten Pericaulom-Stengelpflanzen an die Leitbündel geknüpft sind, nicht bedarf. 
Lippert \& Co. (G. Pätz'sche Buchdr.), Naumburg a. S 


\section{Verlag von Gustav Fischer in Jena.}

Linck, Dr. Gottlob, o. ö. Prof. d. Mineralogie a. d. Univ. Sena, dierende und zum Selbstunterricht. Mit 284 Orig:Figuren i. Text u. 2 farbigen Tafeln. Preis: brosch. 8 Mark, geb. 9 Mlark.

Tabellen zur Gresteinskunde. Für Geologen, Mineralogen, Bergleute, Chemiker, Landwirte und Techniker. Mit 3 Tafeln. 190\%. Preis: 2 Mark:

Regel Dr. Fritz, Prof, der Geographie an der Univ. Jena, Thüringen. Ein geographisches Handbuch. 3 Teile. 1892-9 I. Teil: Das Land. 1. Grenzen. 2. Bodengestalt und Gewässer. 3. Schichtenaufbau und Entstehungsgeschichte. 4. Klima. Mit 1 geologischen Karte, 3 grösseren geologischen Profilen und 40 Textabbildungen. Preis: 8 Mark, geb. 9 Mark. II. Teil: Biogeographie. Erstes Buch: Pflanzen- und Tierverbreitung. Mit 6 Textfiguren. 1894. Preis: 7 Mark. Zweites Buch: Die Bewohner. Mit 94 Textabbildungen. 1895. Preis: 9 Mark. II. Teil geb. 17 Mark 50 Pf. III, T eil: Kulturgeographie. 1896. Preis: 9 Mark, geb. 10 Mark.

Thürringen. Ein landeskundlicher Grundriss. Mit einem Tite 1897. Preis: 4 Mark 50 Pf., geb. 5 Mark.

Rothpletz, A., Das geotektonische Problem der Glarmer Alpen. Mit 11 lithographischen Tafeln und 34 Figuren im Text. Text und Atlas. 1898. Preis : 36 Mark.

Scheibe Dr. Ro, Prof. der Mineralogie an der Kgl. Bergakademie zu Berlin. Geologische Spaziergånge im Thuiringer Wald. Erstes Heft. Preis: 60 Pf.

Solimper Dr. A. F. Wog o. ö. Professor der Botanik an der Universität Basel, Pflanzen- Geographie auf physiologischex Grundlage. Mit 502 als Tafeln oder in den Text gedruckten Abbildungen in Autotypie. 5 Tafeln in Lichtdruck und 4 geographischen Karten. 1898. Preis: brosch. M. 27.-, eleg. in Halbfranz geb. MI. 30.-.

Walthor Joh., Inhaber der Haeckel-Professur für Geologie Walther, und Palaeontologie an der Universität Jena, Einleitung in die Greologie als historische Wissenschafit. 3 Teile. Mit 8 Textabbildungen. Preis: 27 Mlark 50 Pt. I. Teil: Bionomie des Meeres. Beobachtungen über-die marinen Lebensbezirke und Existenzbedingungen. Preis: 6 Mark. - II. Teil: Die Lebensweise der Meerestiere. Beobachtungen über das Leben der geologisch wichtigen Tiere. Preis: 8 Mark 50 Pf. - III. Teil: Lithogenesis der Gegenwart. Beobachtungen über die Bildung der Gesteine an der heutigen Erdoberfäche. Mit 8 Textabb. Preis: 13 Mark.

Demnächst erscheint die zueite vermehrte Auflage von Geologische Heimatskunde von Thüringen. 


\title{
Geologische
}

\section{und Paläontologische Abhandlungen.}

\author{
Herausgegeben \\ von \\ W. Dames und E. Kayser.
}

Neue Folge. Erster Band.

(Der ganzen Reihe fünfter Band.)

Inhalt der bisher erschienenen Hefte:

1. Hol zapfe 1, E., Die cephalopodenführenden

Kalke des unteren Carbon von Erdbach-

Breitscheid bei Herborn. Mit 8 Tafeln. Preis: 16 Mark.

2. Crié, L., Beiträge zur Kenntnis der fossilen Flora einiger Inseln des südpacifischen und indischen Oceans. Mit 10 Tafeln. Preis : 9 Mark.

3. Novák, 0. V'ergleichende Studien an einigen Trilobiten aus dem Hercyn von Bicken, Wildungen, Greifenstein thd Böhmen. Mit 5 Tafeln und 8 Textfiguren. Preis : 10 Mark.

4. Schröder H. Untersuchungen über silurische Cephalopodén. Mit 6 Tafeln und 1 Textfigur.

5. Dames, W., Ueber Zeuglodonten aus Aegypten und die Beziehungen der Archaeoceten zu den übrigen Cetaceen. Mit 7 Tafeln und 1 Textfigur. Preis: 16 Mark.

\section{Neue Folge. Zweiter Band.}

(Der ganzen Reihe sechster Band.)

1. Fu tter er, K., Die oberen Kreidebildungen der Umgebung des Lago di Santa Croce in den Venetianer Alpen. Mit 1 geologischen Karte, 1 Profil-Tafel, 10 Petrefacten-Tafeln und 25 Textfiguren. Preis: 25 Mark

2. Burckhardt, R., Ueber Aepyornis, Mit 4 Tafeln und 2 Textfiguren.

Preis: 6 Mark. Jim b $\overline{0}, \mathrm{~K}$. Beiträge zur Kenntnis der
Fauna der Kreideformation von Hokkaido. Kartenskizze im Text. Preis : 16 Mark.

4. Dames, W., Die Chelonier der norddeutschen Tertiärformation. Mit 4 Tafeln und 3 Textfiguren Preis: 16 Mark.

5. Graf zu Solms-Laubach, H., Ueber Stigmariopsis Grand' Eury. Mit 3 Tafeln und 1 Textfigur. Preis : 7 Mark.

6. Futterer, K., Ueber einige Versteinerungen aus der Kreideformation der karnischen Voralpen. Mit 7 Tafeln und 2 Textfiguren. Preis : 12 Mark.

Neue Folge. Dritter Band. (Der ganzen Reihe siebenter Band.)

1. Jaeke1, 0., Beiträge zur Kenntnis der paläozoischen Crinoiden Deutschlands. Mit 10 Tafeln und 29 Textfiguren.

Preis : 20 Mark

2. Koken, E., Die Reptilien des norddeutschen Wealden. Nachtrag. Mit 4 Tafeln und 1 Textfigur.

Preis: 9 Mark.
3. Steuer, A., Argentinische Jura-Ablagerungen. Ein Beitrag zur Kenntnis der Geologie und Paläontologie der argentinischen Anden. Mit 24 Tafeln, 1 Kartenskizze und 7 Textfiguren. Preis: 40 Mark.

Nene Folge. Viorter Band. Herausgegeben von.

\section{W. Dames und E. Koken.}

(Der ganzen Reihe achter Band.)

1. Kaunhowen, F., Die Gastropodén der Maestrichter Ḱreide. Mit 13 Tafeln.

Preis: 25 Mark.

2. Tornquist, A., Der Dogger am Espinazito-Pass, nebst einer Zusammenstellung der jetzigen Kenntnisse von der argentinischen Juraformation. Mit 10 Tafeln, 1 Profilskizze und 1 Textfigur.

Preis: 22 Mark.

3. Scupin, Hans, Die Spiriferen Deutschlands. Mit 10 Tafeln, 14 Abbildungen im Text und einer schematischen Darstellung. Preis: 28 Mark.

4. Philippi, E., Die Ceratiten des oberen deutschen Muschelkalkes. Mit 21 Tafeln und 19 Abbildungen im Text.

Preis: 40 Mark.

\section{Neue Folge. Fünfter Band. Herausgegeben von \\ E. Koken.}

(Der ganzen Reihe neunter Band.)

1. Frech, F. Geologie der Radstädter Tauern. Mit einer geologischen Karte und 38 Abbildungen im Text.

Preis : 18 Mark.

2. Baltzer, A., Geologie der Umgebung des Iseosees. Mit einer geologischen Karte, einer stratigraphischen Tabelle, 5 Tafeln und 19 Textabbildungen.

Preis: 18 Mark.

3. Schlosser, M., Beiträge zur Kenntnis der Säugetierreste auf den süddeutschen Bohnerzen. Mit 5 Tafeln und 3 Abbildungen im Text.

Preis: 28 Mark.

Neue Folge. Sechster Band.

1. Huene, Fr. v., Uebersicht über die Reptilien der Trias. Mit 9 Tafeln und 78 Textabbildungen. 